hep-th/0311115

MIT-CTP-3440

CALT-68-2462

\title{
Solving Witten's string field theory using the butterfly state
}

\author{
Yuji Okawa \\ Center for Theoretical Physics, Room 6-304 \\ Massachusetts Institute of Technology \\ Cambridge, MA 02139, USA \\ okawa@lns.mit.edu
}

\begin{abstract}
We solve the equation of motion of Witten's cubic open string field theory in a series expansion using the regulated butterfly state. The expansion parameter is given by the regularization parameter of the butterfly state, which can be taken to be arbitrarily small. Unlike the case of level truncation, the equation of motion can be solved for an arbitrary component of the Fock space up to a positive power of the expansion parameter. The energy density of the solution is well defined and remains finite even in the singular butterfly limit, and it gives approximately $68 \%$ of the D25-brane tension for the solution at the leading order. Moreover, it simultaneously solves the equation of motion of vacuum string field theory, providing support for the conjecture at this order. We further improve our ansatz by taking into account next-to-leading terms, and find two numerical solutions which give approximately $88 \%$ and $109 \%$, respectively, of the D25-brane tension for the energy density. These values are interestingly close to those by level truncation at level 2 without gauge fixing studied by Rastelli and Zwiebach and by Ellwood and Taylor.
\end{abstract}




\section{Introduction and summary}

Ever since the pioneering work of Sen and Zwiebach [1], it has been demonstrated that Witten's cubic open string field theory [2] is capable of describing nonperturbative phenomena such as tachyon condensation $[3,4,5,6,7] .{ }^{1}$ The action of Witten's string field theory is given by [2]

$$
S=-\frac{1}{\alpha^{\prime 3} g_{T}^{2}}\left[\frac{1}{2}\left\langle\Psi\left|Q_{B}\right| \Psi\right\rangle+\frac{1}{3}\langle\Psi \mid \Psi * \Psi\rangle\right],
$$

where $Q_{B}$ is the BRST operator and $g_{T}$ is the on-shell three-tachyon coupling constant, and its equation of motion is

$$
Q_{B}|\Psi\rangle+|\Psi * \Psi\rangle=0
$$

The tachyon potential of Witten's string field theory calculated by an approximation scheme called level truncation $[9,1,3,6,7]$ has reproduced the D25-brane tension with impressive precision, providing strong evidence for Sen's conjecture [10, 11]. For instance, if we truncate the string field $|\Psi\rangle$ up to level 2 in the Siegel gauge,

$$
|\Psi\rangle=x c_{1}|0\rangle+2 u c_{-1}|0\rangle+v L_{-2}^{m} c_{1}|0\rangle,
$$

the absolute value of the energy density of the solution obtained by taking variations with respect to the parameters $x, u$, and $v$ is given by approximately $95.9 \%$ of the D25brane tension [1]. However, there are infinitely many other components in the string field so that the solution by level truncation does not solve the equation of motion for a generic component above the truncation level.

On the other hand, various exact solutions of string field theory based on the identity state have been constructed and studied [12, 13, 14, 15, 16, 17, 18, 19, 20, 21, 22, 23, 24]. They exactly solve the equation of motion when it is contracted with any state in the Fock space, but when we try to evaluate the energy density for the solutions, we encounter the notorious singularity coming from the inner product of the identity state with itself.

These previous attempts to solve the equation of motion thus have both good and bad features, which can be summarized in the following way. We expect that the analytic solution $|\Psi\rangle$ corresponding to the tachyon vacuum satisfies the following conditions:

(i) It solves the equation of motion,

$$
\left\langle\phi\left|Q_{B}\right| \Psi\right\rangle+\langle\phi \mid \Psi * \Psi\rangle=0,
$$

\footnotetext{
${ }^{1}$ For a recent review, see [8].
} 
for any state $|\phi\rangle$ in the Fock space.

(ii) The quantities $\left\langle\Psi\left|Q_{B}\right| \Psi\right\rangle$ and $\langle\Psi \mid \Psi * \Psi\rangle$ are well defined, and (ii-a) the equation of motion is satisfied even when contracted with the solution itself:

$$
\left\langle\Psi\left|Q_{B}\right| \Psi\right\rangle+\langle\Psi \mid \Psi * \Psi\rangle=0,
$$

(ii-b) the energy density $\mathcal{E}$ of the solution is given by the D25-brane tension $T_{25}$ as follows $[10,25]$ :

$$
\int d^{26} x \mathcal{E}[\Psi]=\frac{1}{\alpha^{\prime 3} g_{T}^{2}}\left[\frac{1}{2}\left\langle\Psi\left|Q_{B}\right| \Psi\right\rangle+\frac{1}{3}\langle\Psi \mid \Psi * \Psi\rangle\right], \quad \frac{\mathcal{E}[\Psi]}{T_{25}}=-1,
$$

where $T_{25}$ is expressed in terms of $g_{T}$ by $^{2}$

$$
T_{25}=\frac{1}{2 \pi^{2} \alpha^{\prime 3} g_{T}^{2}} .
$$

The solution by level truncation satisfies (ii-a) by construction, and satisfies (ii-b) with very high precision, while the condition (i) is not satisfied for an arbitrary $|\phi\rangle$. The exact solutions based on the identity state satisfy the condition (i), but the quantities $\left\langle\Psi\left|Q_{B}\right| \Psi\right\rangle$ and $\langle\Psi \mid \Psi * \Psi\rangle$ are not well defined so that we cannot even address the questions whether the conditions (ii-a) and (ii-b) are satisfied.

In this paper, we present a new approach to this problem. Our solution satisfies all the above conditions approximately but simultaneously. Our ansatz for a solution takes the form of the regulated butterfly state $[28,29,30,31]$ with an operator inserted at the midpoint of the boundary. ${ }^{3}$

The butterfly state [28] is a star-algebra projector, and the regulated butterfly state $[29,30]$ is its regularization. It is parametrized by $t$ in the range $0 \leq t<1$, and the butterfly state is given by the limit $t \rightarrow 1$. Our solution satisfies the condition (i) up to a positive power of $1-t$. The solution at the leading order is given by choosing a $c$ ghost as the operator to be inserted. If we denote the regulated butterfly state with the operator $\mathcal{O}$ inserted by $\left|B_{t}(\mathcal{O})\right\rangle$, the solution takes the following form:

$$
\left|\Psi^{(0)}\right\rangle=\frac{x}{\sqrt{1-t}}\left|B_{t}(c)\right\rangle
$$

\footnotetext{
${ }^{2}$ See Appendix A of [26] for a check of this relation following Polchinski's conventions in [27].

${ }^{3}$ An analytic framework to solve the equation $\left(L_{0}-1\right)|\Psi\rangle+|\Psi \star \Psi\rangle=0$ using the butterfly state was proposed in [32]. Here $\star$ is the Moyal star product [33, 34, 35, 36, 37, 32, 38], and a closed expression of a formal exact solution has been provided as a perturbative expansion. We use the butterfly state in a different way. Our expansion scheme and lowest-order solution are different from those of [32].
} 
where $x$ is a parameter to be determined. We will show that the equation of motion can be solved up to $O(\sqrt{1-t})$ :

$$
\left\langle\phi\left|Q_{B}\right| \Psi^{(0)}\right\rangle+\left\langle\phi \mid \Psi^{(0)} * \Psi^{(0)}\right\rangle=O(\sqrt{1-t})
$$

for any state $|\phi\rangle$ in the Fock space by this ansatz. The operator $c$ inserted into the regulated butterfly state corresponds to the state $c_{1}|0\rangle$ at level 0 . If we incorporate operators corresponding to states at level 2 into our ansatz

$$
\begin{aligned}
\left|\Psi^{(2)}\right\rangle= & \frac{x}{\sqrt{1-t}}\left|B_{t}(c)\right\rangle \\
& +\sqrt{1-t}\left[u\left|B_{t}\left(\partial^{2} c\right)\right\rangle+v\left|B_{t}\left(c T^{m}\right)\right\rangle+w\left|B_{t}(: b c \partial c:)\right\rangle\right],
\end{aligned}
$$

where $x, u, v$, and $w$ are parameters to be determined, it turns out that the equation of motion can be solved up to a higher power of $1-t$ :

$$
\left\langle\phi\left|Q_{B}\right| \Psi^{(2)}\right\rangle+\left\langle\phi \mid \Psi^{(2)} * \Psi^{(2)}\right\rangle=O\left((1-t)^{\frac{3}{2}}\right)
$$

for any state $|\phi\rangle$ in the Fock space. We find one analytic solution in the form of $\left|\Psi^{(0)}\right\rangle$ and two numerical solutions $\left|\Psi_{1}^{(2)}\right\rangle$ and $\left|\Psi_{2}^{(2)}\right\rangle$ in the form of $\left|\Psi^{(2)}\right\rangle$. Therefore, the condition (i) can be satisfied with high precision if we choose $t$ to be close to 1 . Furthermore, it turns out that the inner products appearing in the condition (ii) are well defined for our solutions. In order to discuss the condition (ii-a), let us define the ratio $\mathcal{R}[\Psi]$ for a string field $|\Psi\rangle$ by

$$
\mathcal{R}[\Psi] \equiv \frac{\left\langle\Psi\left|Q_{B}\right| \Psi\right\rangle}{\langle\Psi \mid \Psi * \Psi\rangle}
$$

The condition (ii-a) is satisfied when $\mathcal{R}[\Psi]=-1$. For the leading-order solution $\left|\Psi^{(0)}\right\rangle$, $\mathcal{R}\left[\Psi^{(0)}\right]$ and $\mathcal{E}\left[\Psi^{(0)}\right] / T_{25}$ are given by

$$
\begin{aligned}
& \lim _{t \rightarrow 1} \mathcal{R}\left[\Psi^{(0)}\right]=-2^{\frac{15}{2}} \cdot 3^{-\frac{19}{4}} \simeq-0.9804 \\
& \lim _{t \rightarrow 1} \frac{\mathcal{E}\left[\Psi^{(0)}\right]}{T_{25}}=2 \pi^{2}\left(-2^{-4} \cdot 3^{\frac{1}{2}}+2^{-\frac{21}{2}} \cdot 3^{\frac{17}{4}}\right) \simeq-0.6838
\end{aligned}
$$

The condition (ii-a) is satisfied with precision of approximately $98 \%$, and the absolute value of the energy density gives approximately $68 \%$ of the D25-brane tension. These values are improved for the solutions at the next-to-leading order:

$$
\begin{array}{ll}
\lim _{t \rightarrow 1} \mathcal{R}\left[\Psi_{1}^{(2)}\right] \simeq-0.9989, & \lim _{t \rightarrow 1} \frac{\mathcal{E}\left[\Psi_{1}^{(2)}\right]}{T_{25}} \simeq-0.8826 \\
\lim _{t \rightarrow 1} \mathcal{R}\left[\Psi_{2}^{(2)}\right] \simeq-0.9981, & \lim _{t \rightarrow 1} \frac{\mathcal{E}\left[\Psi_{2}^{(2)}\right]}{T_{25}} \simeq-1.0898
\end{array}
$$


The condition (ii-a) is satisfied with better precision than $99.8 \%$, and the values of the energy density for these solutions are approximately $88 \%$ and $109 \%$, respectively, of the D25-brane tension.

It is not surprising that there are more than one solution at this order since we do not fix gauge. There are also two solutions at level 2 in level truncation without gauge fixing studied by Rastelli and Zwiebach [39] and by Ellwood and Taylor [40]. In fact, the values of the ratio $\mathcal{E} / T_{25}$ for our solutions are interestingly close to those from the level-truncation analysis without gauge fixing, which are $\mathcal{E} / T_{25}=-4096 \pi^{2} / 59049 \simeq$ -0.6846 for the solution at level 0 , and $\mathcal{E} / T_{25} \simeq-0.8802,-1.0778$ for the two solutions at level $2[40]$, as compared in Table 1.

Table 1: Comparison of $-\mathcal{E} / T_{25}$ with level truncation without gauge fixing.

\begin{tabular}{|c|c||c|c|}
\hline Our solutions & $-\mathcal{E} / T_{25}$ & Level truncation & $-\mathcal{E} / T_{25}$ \\
\hline$\Psi^{(0)}$ & 0.6838 & level 0 & 0.6846 \\
\hline$\Psi^{(2)}$ & $0.8826,1.0898$ & level 2 & $0.8802,1.0778$ \\
\hline
\end{tabular}

There is another interesting aspect of our solution. Our leading-order solution $\left|\Psi^{(0)}\right\rangle$ satisfies not only the equation of motion of Witten's string field theory but also that of vacuum string field theory $[41,42,43]$ at the leading order,

$$
-\left\langle\phi|\mathcal{Q}| \Psi^{(0)}\right\rangle+\left\langle\phi \mid \Psi^{(0)} * \Psi^{(0)}\right\rangle=O(\sqrt{1-t})
$$

when $\mathcal{Q}$ is given by the midpoint $c$-ghost insertion with an appropriate normalization [28]. This can be regarded as support for the vacuum string field theory conjecture at this order. It was shown [28] that a star-algebra projector in the twisted ghost conformal field theory solves the equation of motion of vacuum string field theory in the singular limit, but our solution is different from the twisted butterfly state and it is a new solution to vacuum string field theory. Note also that the twisted butterfly state does not solve the equation of motion of Witten's string field theory even at the leading order.

It is not difficult to see how our ansatz works. Actually, we can use any regulated star-algebra projector instead of the regulated butterfly state. ${ }^{4}$ The star product of the regulated star-algebra projector with itself approximately reproduces itself. In the process of gluing, the midpoint of the boundary of each projector, where an operator is inserted, is mapped to a point which is very close to the midpoint of the boundary of

\footnotetext{
${ }^{4}$ We are aware that this idea at the leading order occurred to many others independently including I. Ellwood, L. Rastelli, M. Schnabl, A. Sen, and B. Zwiebach.
} 
the glued surface state. Therefore, the two operators from each of the projector can be replaced by the leading term of their operator product expansion (OPE). On the other hand, the only effect of the BRST operator $Q_{B}$ acting on the regulated projector with an operator insertion is to make the BRST transformation of the inserted operator. If we take the operator to be a $c$ ghost, the leading term of the OPE is given by $c \partial c$,

$$
c(z) c(w)=-(z-w) c \partial c(w)+O\left((z-w)^{2}\right),
$$

and the BRST transformation of the $c$ ghost is also $c \partial c$ :

$$
Q_{B} \cdot c(w)=\oint \frac{d z}{2 \pi i} j_{B}(z) c(w)=c \partial c(w)
$$

where $j_{B}(z)$ is the BRST current. Therefore, the equation of motion of Witten's string field theory, $Q_{B}|\Psi\rangle+|\Psi * \Psi\rangle=0$, can be satisfied at the leading order since the two terms are the same surface state with $c \partial c$ inserted at the midpoint of the boundary.

Similarly, it can also be easily seen how our ansatz solves the equation of motion of vacuum string field theory at the leading order. When we contract a regulated staralgebra projector with a state in the Fock state, the open-string midpoint approaches the midpoint of the boundary $[44,30]$. Therefore, the two $c$ ghosts, one from the kinetic operator $\mathcal{Q}$ inserted at the open-string midpoint and the other one inserted at the midpoint of the boundary, can be replaced by the leading term of the OPE, which is again given by $c \partial c$.

The organization of the rest of the paper is as follows. In Section 2, the leadingorder solution $\left|\Psi^{(0)}\right\rangle$ is constructed and its properties are studied. In Section 3, it is shown that the leading-order solution with an opposite sign, $-\left|\Psi^{(0)}\right\rangle$, also solves the equation of motion of vacuum string field theory at the leading order. In Section 4, the solutions $\left|\Psi_{1}^{(2)}\right\rangle$ and $\left|\Psi_{2}^{(2)}\right\rangle$ are constructed by taking into account next-to-leading terms, and the energy density is computed for these solutions. Section 5 is devoted to discussion. Our conventions and terminology on the conformal field theory (CFT) formulation of string field theory $[45,46]$ are summarized in Appendix A. An explicit expression of the quantity $\left\langle\Psi^{(2)} \mid \Psi^{(2)} * \Psi^{(2)}\right\rangle$ for an arbitrary $t$ is presented in Appendix B.

\section{Solution at the leading order}

\subsection{Regulated butterfly state}

The regulated butterfly state $\left|B_{t}\right\rangle$ labeled by $t$ in the range $0 \leq t<1[29,30]$ is defined by

$$
\left\langle\phi \mid B_{t}\right\rangle=\left\langle f_{t} \circ \phi(0)\right\rangle
$$


for any state $|\phi\rangle$ in the Fock space, where

$$
f_{t}(\xi)=\frac{\xi}{\sqrt{1+t^{2} \xi^{2}}}
$$

All CFT correlation functions in this paper are evaluated on an upper-half plane, and we use the doubling trick. The butterfly state $|B\rangle$ is given by the regulated butterfly state in the limit $t \rightarrow 1$. It is a singular state like other star-algebra projectors such as the sliver state [47]. The singularity can be seen, for example, by the fact that the open-string midpoint $f_{t}(i)$ reaches the boundary in the limit $t \rightarrow 1$. However, an inner product of the regulated butterfly state with a state $|\phi\rangle$ in the Fock space is well defined even in the limit $t \rightarrow 1$ and is given by

$$
\langle\phi \mid B\rangle=\lim _{t \rightarrow 1}\left\langle\phi \mid B_{t}\right\rangle=\left\langle f_{B} \circ \phi(0)\right\rangle
$$

where

$$
f_{B}(\xi)=\frac{\xi}{\sqrt{1+\xi^{2}}}
$$

The regulated butterfly state reduces to the $S L(2, R)$-invariant vacuum $|0\rangle$ when $t=0$.

We can use different conformal transformations to represent the same surface state. For example, the regulated butterfly state can also be represented as

$$
\left\langle\phi \mid B_{t}\right\rangle=\left\langle h_{t} \circ \phi(0)\right\rangle
$$

for any state $|\phi\rangle$ in the Fock space, where $h_{t}(\xi)$ is the following conformal transformation with a parameter $p$ :

$$
h_{t}(\xi)=\frac{\xi}{\xi+p \sqrt{1+t^{2} \xi^{2}}} .
$$

The conformal transformation $h_{t}(\xi)$ is related to $f_{t}(\xi)$ by an $S L(2, R)$ transformation $z /(z+p)$ which maps the infinity to 1 . The conformal transformations with different values of $p$ for (2.6) are all equivalent and define the same state. This representation will be useful when there is an operator insertion at the infinity in the representation in terms of $f_{t}(\xi)$. In this representation, the inner product $\langle\phi \mid B\rangle$ in the butterfly limit is given by

$$
\langle\phi \mid B\rangle=\left\langle h_{B} \circ \phi(0)\right\rangle
$$

where

$$
h_{B}(\xi)=\frac{\xi}{\xi+p \sqrt{1+\xi^{2}}} .
$$

Another useful representation of the regulated butterfly state $\left|B_{t}\right\rangle$ is given by making an inversion $I(z)=-1 / z$ to the conformal transformation $f_{t}(\xi)$ :

$$
I \circ f_{t}(\xi)=-\frac{\sqrt{1+t^{2} \xi^{2}}}{\xi} .
$$


Using this conformal transformation, the regulated butterfly state is represented as

$$
\left\langle\phi \mid B_{t}\right\rangle=\left\langle I \circ f_{t} \circ \phi(0)\right\rangle \text {. }
$$

The coordinate $z^{\prime}=I \circ f_{t}(\xi)$ is related to the coordinate $z=h_{t}(\xi)$ by

$$
z^{\prime}=\frac{z-1}{p z}, \quad z=\frac{1}{1-p z^{\prime}} .
$$

Finally, the regulated butterfly state has a simple representation in the operator formalism $[48,49,50,51,52]$ in terms of a single Virasoro generator [29, 30]. It is given by

$$
\left|B_{t}\right\rangle=\exp \left(-\frac{t^{2}}{2} L_{-2}\right)|0\rangle \text {. }
$$

The conformal transformation associated with the star product of the regulated butterfly state $\left|B_{t} * B_{t}\right\rangle$ was derived by Schnabl [29]. After an appropriate rescaling of Schnabl's expression discussed in [31], the state $\left|B_{t} * B_{t}\right\rangle$ is represented as

$$
\left\langle\phi \mid B_{t} * B_{t}\right\rangle=\left\langle\tilde{f}_{t} \circ \phi(0)\right\rangle,
$$

where

$$
\tilde{f}_{t}(\xi)=\sqrt{\frac{3}{4} \frac{9-a^{2}}{\left(1-a^{2}\right)\left(a^{2}+3\right)}\left[\tan ^{2}\left(\frac{2}{3} \arctan \sqrt{\frac{\xi^{2}+t^{2}}{1+t^{2} \xi^{2}}}\right)-\frac{a^{2}}{3}\right]}
$$

with

$$
a \equiv \sqrt{3} \tan \left(\frac{2}{3} \arctan t\right) .
$$

It can be easily verified that $\widetilde{f}_{t}(\xi)$ reduces to $f_{B}(\xi)$ in the limit $t \rightarrow 1$ :

$$
\tilde{f}_{t}(\xi)=f_{B}(\xi)+O(1-t) .
$$

Therefore,

$$
\left\langle\phi \mid B_{t} * B_{t}\right\rangle=\left\langle\phi \mid B_{t}\right\rangle+O(1-t) .
$$

This shows that the butterfly state $|B\rangle$ is a star-algebra projector. The relation between the coordinate $z=\widetilde{f}_{t}(\xi)$ of $\left\langle\phi \mid B_{t} * B_{t}\right\rangle$ and the coordinate $z^{\prime}=I \circ f_{t}(\xi)$ of $\left\langle\phi \mid B_{t}\right\rangle$ before gluing was derived in [31]. It is given by

$$
z^{\prime 2}=\frac{\left(1+t^{2}\right)\left(1+d^{2} z^{2}\right)^{\frac{3}{2}}+\left(1-t^{2}\right)\left(1-\frac{4 \beta^{2}}{d^{2}} z^{2}\right)}{\left(1+d^{2} z^{2}\right)^{\frac{3}{2}}-\left(1-\frac{4 \beta^{2}}{d^{2}} z^{2}\right)},
$$

where

$$
\beta=\frac{2}{9-a^{2}} \sqrt{\left(1-a^{2}\right)\left(a^{2}+3\right)}, \quad d=2 \sqrt{\frac{1-a^{2}}{9-a^{2}}} .
$$

The midpoint of the boundary $z^{\prime}=0$ is mapped to $z= \pm 1 / \beta$, and the open-string midpoint $z^{\prime}=i$ is mapped to $z=i / d[30,31]$. 


\subsection{Solving the equation of motion at the leading order}

Our ansatz for a solution to Witten's string field theory takes the form of the regulated butterfly state with an operator insertion at the midpoint of the boundary. Let us define $\left|B_{t}(\mathcal{O})\right\rangle$ by

$$
\left\langle\phi \mid B_{t}(\mathcal{O})\right\rangle=\left\langle I \circ f_{t} \circ \phi(0) \mathcal{O}(0)\right\rangle
$$

for any state $|\phi\rangle$ in the Fock space. As in the case of the regulated butterfly state $\left|B_{t}\right\rangle$, the state $\left|B_{t}(\mathcal{O})\right\rangle$ itself becomes singular in the limit $t \rightarrow 1$, but its inner product with a state $|\phi\rangle$ in the Fock space has a finite limit, which we denote by $\langle\phi \mid B(\mathcal{O})\rangle$ :

$$
\langle\phi \mid B(\mathcal{O})\rangle=\lim _{t \rightarrow 1}\left\langle\phi \mid B_{t}(\mathcal{O})\right\rangle=\left\langle I \circ f_{B} \circ \phi(0) \mathcal{O}(0)\right\rangle .
$$

When the operator $\mathcal{O}$ is a $c$ ghost, the representation of the state $\left|B_{t}(c)\right\rangle$ using $h_{t}(\xi)$ is given by

$$
\left\langle\phi \mid B_{t}(c)\right\rangle=\left\langle I \circ f_{t} \circ \phi(0) c(0)\right\rangle=\frac{1}{p}\left\langle h_{t} \circ \phi(0) c(1)\right\rangle
$$

for any state $|\phi\rangle$ in the Fock space. As we mentioned in the Introduction, the state $\left|B_{t}(c)\right\rangle$ with an appropriate normalization solves the equation of motion of Witten's string field theory up to $O(\sqrt{1-t})$. In what follows we will use $|\phi\rangle$ to denote a state in the Fock space.

Let us compute $\left\langle\phi\left|Q_{B}\right| B_{t}(c)\right\rangle$ and $\left\langle\phi \mid B_{t}(c) * B_{t}(c)\right\rangle$. The quantity $\left\langle\phi\left|Q_{B}\right| B_{t}(c)\right\rangle$ is given by

$$
\left\langle\phi\left|Q_{B}\right| B_{t}(c)\right\rangle=\frac{1}{p}\left\langle h_{t} \circ \phi(0) Q_{B} \cdot c(1)\right\rangle,
$$

where $Q_{B} \cdot \mathcal{O}(w)$ denotes the BRST transformation of the operator $\mathcal{O}(w)$ defined by

$$
Q_{B} \cdot \mathcal{O}(w)=\oint \frac{d z}{2 \pi i} j_{B}(z) \mathcal{O}(w),
$$

where the contour of the integral encircles $w$ counterclockwise. The BRST current $j_{B}(z)$ here is defined by

$$
j_{B}(z)=c T^{m}(z)+: b c \partial c:(z)+\frac{3}{2} \partial^{2} c(z),
$$

where $T^{m}(z)$ denotes the energy-momentum tensor of the matter sector and : $\mathcal{O}:(z)$ denotes the normal ordering of the operator $\mathcal{O}(z)$. Since the OPE between $j_{B}$ and $c$ is

$$
j_{B}(z) c(w) \sim \frac{1}{z-w} c \partial c(w),
$$

the BRST transformation of $c(w)$ is given by

$$
Q_{B} \cdot c(w)=c \partial c(w) .
$$


Therefore, we find

$$
\left\langle\phi\left|Q_{B}\right| B_{t}(c)\right\rangle=\frac{1}{p}\left\langle h_{t} \circ \phi(0) c \partial c(1)\right\rangle
$$

or, in other words,

$$
\left\langle\phi\left|Q_{B}\right| B_{t}(c)\right\rangle=\left\langle\phi \mid B_{t}(c \partial c)\right\rangle .
$$

As is clear from this derivation, $Q_{B}\left|B_{t}(\mathcal{O})\right\rangle$ is in general given by

$$
Q_{B}\left|B_{t}(\mathcal{O})\right\rangle=\left|B_{t}\left(Q_{B} \cdot \mathcal{O}\right)\right\rangle .
$$

The only $t$ dependence of (2.28) is coming from that of the conformal transformation $h_{t}(\xi)$. Since

$$
h_{t}(\xi)=h_{B}(\xi)+O(1-t),
$$

the leading term of the expansion in $1-t$ is given by

$$
\left\langle\phi\left|Q_{B}\right| B_{t}(c)\right\rangle=\frac{1}{p}\left\langle h_{B} \circ \phi(0) c \partial c(1)\right\rangle+O(1-t),
$$

or

$$
\left\langle\phi\left|Q_{B}\right| B_{t}(c)\right\rangle=\langle\phi \mid B(c \partial c)\rangle+O(1-t) .
$$

In order to compute the other quantity $\left\langle\phi \mid B_{t}(c) * B_{t}(c)\right\rangle$, we need to know how the $c$ ghosts are mapped to the glued surface. In the coordinate $z^{\prime}=I \circ f_{t}(\xi)$ representing $\left\langle\phi \mid B_{t}(c)\right\rangle$, the $c$ ghost is inserted at $z^{\prime}=0$. This point is mapped to $z= \pm 1 / \beta$ in the coordinate $z=\widetilde{f}_{t}(\xi)$ of $\left\langle\phi \mid B_{t} * B_{t}\right\rangle$. The derivatives $d z^{\prime} / d z$ at these points were computed from (2.18) in [31] and are given by

$$
\left.\frac{d z^{\prime}}{d z}\right|_{z= \pm \frac{1}{\beta}}=\sqrt{\frac{\left(1-t^{4}\right)\left(1-a^{2}\right)\left(3+a^{2}\right)}{48}} .
$$

Therefore, the $c$ ghost is mapped from the $z^{\prime}$ coordinate to the $z$ coordinate as follows:

$$
c(0) \rightarrow \sqrt{\frac{\left(1-t^{4}\right)\left(1-a^{2}\right)\left(3+a^{2}\right)}{48}} c\left( \pm \frac{1}{\beta}\right) .
$$

Since

$$
\beta=\frac{\sqrt{2}}{3^{\frac{3}{4}}} \sqrt{1-t}+O\left((1-t)^{\frac{3}{2}}\right),
$$

the two $c$ ghosts are sent to the infinity in the limit $t \rightarrow 1$. It is therefore convenient to make an $S L(2, R)$ transformation to bring the infinity to a finite point to study the limit $t \rightarrow 1$. If we make the conformal map $z /(z+p)$, the $c$ ghosts are further transformed as

$$
c\left( \pm \frac{1}{\beta}\right) \rightarrow \frac{(1 \pm p \beta)^{2}}{p \beta^{2}} c\left(\frac{1}{1 \pm p \beta}\right) .
$$


Therefore, the quantity $\left\langle\phi \mid B_{t}(c) * B_{t}(c)\right\rangle$ is given by

$$
\begin{aligned}
\left\langle\phi \mid B_{t}(c) * B_{t}(c)\right\rangle= & \frac{\left(1-t^{4}\right)\left(1-a^{2}\right)\left(3+a^{2}\right)}{48} \frac{\left(1-p^{2} \beta^{2}\right)^{2}}{p^{2} \beta^{4}} \\
& \times\left\langle\widetilde{h}_{t} \circ \phi(0) c\left(\frac{1}{1+p \beta}\right) c\left(\frac{1}{1-p \beta}\right)\right\rangle,
\end{aligned}
$$

where

$$
\widetilde{h}_{t}(\xi)=\frac{\tilde{f}_{t}(\xi)}{\tilde{f}_{t}(\xi)+p} .
$$

In the limit $t \rightarrow 1$, the two $c$ ghosts approach the point $z=1$ so that they can be replaced by the leading term of their OPE, which is given by

$$
c\left(\frac{1}{1+p \beta}\right) c\left(\frac{1}{1-p \beta}\right)=2 p \beta c \partial c(1)+O\left(\beta^{3}\right) .
$$

Note that terms of $O\left(\beta^{2}\right)$ cancel. The leading term of $\left\langle\phi \mid B_{t}(c) * B_{t}(c)\right\rangle$ in the limit $t \rightarrow 1$ is given by

$$
\begin{aligned}
\left\langle\phi \mid B_{t}(c) * B_{t}(c)\right\rangle & =\frac{4 \sqrt{2}}{3^{\frac{1}{4}}} \frac{\sqrt{1-t}}{p}\left\langle h_{B} \circ \phi(0) c \partial c(1)\right\rangle+O\left((1-t)^{\frac{3}{2}}\right) \\
& =\frac{4 \sqrt{2}}{3^{\frac{1}{4}}} \sqrt{1-t}\langle\phi \mid B(c \partial c)\rangle+O\left((1-t)^{\frac{3}{2}}\right) .
\end{aligned}
$$

Therefore, the leading term of $\left\langle\phi \mid B_{t}(c) * B_{t}(c)\right\rangle$ is proportional to that of $\left\langle\phi\left|Q_{B}\right| B_{t}(c)\right\rangle$. If we define

$$
\left|\Psi^{(0)}\right\rangle=-\frac{3^{\frac{1}{4}}}{4 \sqrt{2}} \frac{1}{\sqrt{1-t}}\left|B_{t}(c)\right\rangle,
$$

then the state $\left|\Psi^{(0)}\right\rangle$ solves the equation of motion of Witten's string field theory up to $O(\sqrt{1-t})$ :

$$
\left\langle\phi\left|Q_{B}\right| \Psi^{(0)}\right\rangle+\left\langle\phi \mid \Psi^{(0)} * \Psi^{(0)}\right\rangle=O(\sqrt{1-t})
$$

for any state $|\phi\rangle$ in the Fock space. If we take the limit $t \rightarrow 1$, the state $\left|\Psi^{(0)}\right\rangle$ formally solves the equation of motion exactly. However, the regulated butterfly state $\left|B_{t}\right\rangle$ becomes singular and the coefficient diverges as $1 / \sqrt{1-t}$ in the limit so that we do not intend to take the strict $t \rightarrow 1$ limit. On the other hand, the state $\left|\Psi^{(0)}\right\rangle$ is well defined as long as $1-t$ is finite. If we choose $t$ to be 0.9999 , for example, the equation of motion is solved with fairly good precision for any state $|\phi\rangle$ in the Fock space.

\subsection{Energy density at the leading order}

Let us next evaluate the energy density of the solution. We need to compute the inner products $\left\langle B_{t}(c)\left|Q_{B}\right| B_{t}(c)\right\rangle$ and $\left\langle B_{t}(c) \mid B_{t}(c) * B_{t}(c)\right\rangle$, but similar computations have been done in [31] so that we can make use of the results in [31]. 
When we deal with the star multiplication of the regulated butterfly state, it is convenient to use the $\hat{z}$ coordinate [30] defined by

$$
\hat{z}=\arctan \xi \text {. }
$$

In the $\hat{z}$ coordinate, either of the left and right halves of the open string of the regulated butterfly state is mapped to a semi-infinite line parallel to the imaginary axis in the upper-half plane so that gluing can be made simply by translation. We glue two or three regulated butterfly states for $\left\langle B_{t}(c)\left|Q_{B}\right| B_{t}(c)\right\rangle$ or $\left\langle B_{t}(c) \mid B_{t}(c) * B_{t}(c)\right\rangle$, respectively, in this way. We then map the resulting surface to an upper-half plane by a conformal transformation. Let us denote the coordinate of the upper-half plane by $z$. The relation between the $z$ coordinate and the coordinate $z^{\prime}=I \circ f_{t}(\xi)$ before gluing was derived in [31]. In the case of $\left\langle B_{t}(c)\left|Q_{B}\right| B_{t}(c)\right\rangle$, it is given by

$$
\frac{4\left(z^{\prime 2}-t^{2}\right)}{\left[z^{\prime 2}-\left(1+t^{2}\right)\right]^{2}}=\frac{\left(1-z^{2}\right)^{2}-4 q^{2} z^{2}}{4\left(1+q^{2}\right) z^{2}}
$$

where

$$
q=\frac{2 t}{1-t^{2}}
$$

and the relation for $\left\langle B_{t}(c) \mid B_{t}(c) * B_{t}(c)\right\rangle$ is given as follows:

$$
\frac{4\left(z^{\prime 2}-t^{2}\right)}{\left[z^{\prime 2}-\left(1+t^{2}\right)\right]^{2}}=\frac{z^{2}\left(z^{2}-3\right)^{2}-q^{2}\left(1-3 z^{2}\right)^{2}}{\left(1+q^{2}\right)\left(1-3 z^{2}\right)^{2}} \text {. }
$$

The $c$ ghost at $z^{\prime}=0$ is mapped to

$$
c(0) \rightarrow \frac{\sqrt{1-t^{4}}}{2} c( \pm 1)
$$

in the $z$ coordinate of $\left\langle B_{t}(c)\left|Q_{B}\right| B_{t}(c)\right\rangle$, and to

$$
c(0) \rightarrow \frac{3}{8} \sqrt{1-t^{4}} c(-\sqrt{3}), \quad \frac{3}{2} \sqrt{1-t^{4}} c(0), \quad \frac{3}{8} \sqrt{1-t^{4}} c(\sqrt{3})
$$

in the $z$ coordinate of $\left\langle B_{t}(c) \mid B_{t}(c) * B_{t}(c)\right\rangle$. The inner product $\left\langle B_{t}(c)\left|Q_{B}\right| B_{t}(c)\right\rangle$ is therefore given by

$$
\begin{aligned}
\left\langle B_{t}(c)\left|Q_{B}\right| B_{t}(c)\right\rangle_{\text {density }} & =\frac{1-t^{4}}{4}\left\langle c(-1) Q_{B} \cdot c(1)\right\rangle_{\text {density }} \\
& =\frac{1-t^{4}}{4}\langle c(-1) c \partial c(1)\rangle_{\text {density }}=-\left(1-t^{4}\right),
\end{aligned}
$$

where the subscript density denotes that the quantity is divided by the volume factor of space-time. We use this notation for both inner products of string fields and CFT 
correlation functions. Similarly, $\left\langle B_{t}(c) \mid B_{t}(c) * B_{t}(c)\right\rangle$ is computed as follows:

$$
\begin{aligned}
\left\langle B_{t}(c) \mid B_{t}(c) * B_{t}(c)\right\rangle_{\text {density }} & =\frac{27}{128}\left(1-t^{4}\right)^{\frac{3}{2}}\langle c(-\sqrt{3}) c(0) c(\sqrt{3})\rangle_{\text {density }} \\
& =-\left(\frac{3 \sqrt{3}}{4}\right)^{3}\left(1-t^{4}\right)^{\frac{3}{2}}
\end{aligned}
$$

In both cases, the inner product cancels the singularity coming from the normalization factor so that $\left\langle\Psi^{(0)}\left|Q_{B}\right| \Psi^{(0)}\right\rangle$ and $\left\langle\Psi^{(0)} \mid \Psi^{(0)} * \Psi^{(0)}\right\rangle$ have a finite density in the limit $t \rightarrow 1$. Since $\left\langle\Psi^{(0)}\left|Q_{B}\right| \Psi^{(0)}\right\rangle$ and $\left\langle\Psi^{(0)} \mid \Psi^{(0)} * \Psi^{(0)}\right\rangle$ are well defined, we can discuss the equation of motion contracted with the solution itself. Let us compute the ratio $\mathcal{R}\left[\Psi^{(0)}\right]$ defined in (1.12) in the limit $t \rightarrow 1:$

$$
\lim _{t \rightarrow 1} \mathcal{R}\left[\Psi^{(0)}\right]=\lim _{t \rightarrow 1} \frac{\left\langle\Psi^{(0)}\left|Q_{B}\right| \Psi^{(0)}\right\rangle}{\left\langle\Psi^{(0)} \mid \Psi^{(0)} * \Psi^{(0)}\right\rangle}=-2^{\frac{15}{2}} \cdot 3^{-\frac{19}{4}} \simeq-0.9804
$$

The ratio is not exactly -1 , but rather close to -1 so that the equation of motion is satisfied with fairly good precision even when it is contracted with the solution itself. In other words, the solution $\left|\Psi^{(0)}\right\rangle$ satisfies the condition (ii-a) in the Introduction with good precision.

Let us next evaluate the energy density of the solution $\mathcal{E}$ defined by

$$
\mathcal{E}[\Psi]=\frac{1}{\alpha^{\prime 3} g_{T}^{2}}\left[\frac{1}{2}\left\langle\Psi\left|Q_{B}\right| \Psi\right\rangle_{\text {density }}+\frac{1}{3}\langle\Psi \mid \Psi * \Psi\rangle_{\text {density }}\right] .
$$

The energy density of the solution $\mathcal{E}\left[\Psi^{(0)}\right]$ divided by the D25-brane tension $T_{25}$ in the limit $t \rightarrow 1$ is given by

$$
\lim _{t \rightarrow 1} \frac{\mathcal{E}\left[\Psi^{(0)}\right]}{T_{25}}=2 \pi^{2}\left(-2^{-4} \cdot 3^{\frac{1}{2}}+2^{-\frac{21}{2}} \cdot 3^{\frac{17}{4}}\right) \simeq-0.6838 .
$$

Therefore, the absolute value of the energy density for the solution $\left|\Psi^{(0)}\right\rangle$ gives approximately $68 \%$ of the D25-brane tension. This value is very close to that obtained by level truncation at level $0[1], \mathcal{E} / T_{25}=-4096 \pi^{2} / 59049 \simeq-0.6846$, but not exactly the same. We conclude that our solution $\left|\Psi^{(0)}\right\rangle$ at the leading order is as good as the level-0 solution by level truncation concerning the condition (ii-b) in the Introduction.

We have seen that our solution $\left|\Psi^{(0)}\right\rangle$ approximately satisfies the condition (ii-a), but there are no a priori reasons to expect that this should be the case. As can be seen from the expression (2.51), the $c$ ghosts can no longer be replaced by $c \partial c$ so that the mechanism responsible for the condition (i) does not work when the equation of motion is contracted with the solution itself. There are also no a priori reasons to expect that the energy density of our solution $\left|\Psi^{(0)}\right\rangle$ is of the same order as the D25-brane tension just like there are no a priori reasons to expect that the ordinary level-truncation approximation should work. 


\subsection{Comparison with a solution by a variational method}

We can perform an analysis similar to level truncation by truncating the string field to the single mode $\left|B_{t}(c)\right\rangle$,

$$
|\Psi\rangle=\frac{\tilde{x}}{\sqrt{1-t^{4}}}\left|B_{t}(c)\right\rangle
$$

and by taking a variation with respect to the parameter $\tilde{x}$. It reduces to the ordinary level-truncation analysis at level 0 when we set $t=0$. The energy density divided by the D25-brane tension is given by

$$
\frac{\mathcal{E}(\tilde{x})}{T_{25}}=2 \pi^{2}\left[-\frac{1}{2} \tilde{x}^{2}-\frac{1}{3}\left(\frac{3 \sqrt{3}}{4}\right)^{3} \tilde{x}^{3}\right] .
$$

This is independent of $t$ and is exactly the same as in the case of the ordinary truncation to $|\Psi\rangle=\tilde{x} c_{1}|0\rangle$. Therefore, the critical value $\tilde{x}_{c}$ determined by the condition

$$
\left.\frac{d}{d \tilde{x}} \mathcal{E}(\tilde{x})\right|_{\tilde{x}=\tilde{x}_{c}}=0
$$

and $\mathcal{E}\left(\tilde{x}_{c}\right) / T_{25}$ are also the same as in the case of the ordinary level truncation:

$$
\tilde{x}_{c}=-\left(\frac{4}{3 \sqrt{3}}\right)^{3}, \quad \frac{\mathcal{E}\left(\tilde{x}_{c}\right)}{T_{25}}=-\frac{4096 \pi^{2}}{59049} \simeq-0.6846 .
$$

The string field with $\tilde{x}=\tilde{x}_{c}$,

$$
\left|\widetilde{\Psi}^{(0)}\right\rangle=-\left(\frac{4}{3 \sqrt{3}}\right)^{3} \frac{1}{\sqrt{1-t^{4}}}\left|B_{t}(c)\right\rangle
$$

by construction satisfies the condition (ii-a),

$$
\left\langle\widetilde{\Psi}^{(0)}\left|Q_{B}\right| \widetilde{\Psi}^{(0)}\right\rangle+\left\langle\widetilde{\Psi}^{(0)} \mid \widetilde{\Psi}^{(0)} * \widetilde{\Psi}^{(0)}\right\rangle=0
$$

but does not solve the equation of motion when it is contracted with a generic state in the Fock space. The normalization of our solution $\left|\Psi^{(0)}\right\rangle$ is in fact numerically close to that of $\left|\widetilde{\Psi}^{(0)}\right\rangle$ in the limit $t \rightarrow 1$ :

$$
\lim _{t \rightarrow 1} \frac{\left|\widetilde{\Psi}^{(0)}\right\rangle}{\left|\Psi^{(0)}\right\rangle}=2^{\frac{15}{2}} \cdot 3^{-\frac{19}{4}} \simeq 0.9804 .
$$

This is why our solution $\left|\Psi^{(0)}\right\rangle$ approximately satisfies the equation $\left\langle\Psi^{(0)}\left|Q_{B}\right| \Psi^{(0)}\right\rangle+$ $\left\langle\Psi^{(0)} \mid \Psi^{(0)} * \Psi^{(0)}\right\rangle=0$, and approximately reproduces the value of the energy density obtained by the ordinary level truncation at level 0 , but again we do not know why this is the case. 


\section{Solution to both Witten's and vacuum string field theories}

If we expand the action of Witten's string field theory (1.1) around the solution $\left|\Psi_{0}\right\rangle$ corresponding to the tachyon vacuum, the resulting action will take the same form except for the kinetic operator:

$$
S=S_{0}-\frac{1}{\alpha^{\prime 3} g_{T}^{2}}\left[\frac{1}{2}\langle\Psi|\mathcal{Q}| \Psi\rangle+\frac{1}{3}\langle\Psi \mid \Psi * \Psi\rangle\right]
$$

where $S_{0}$ is the value of the action for $\left|\Psi_{0}\right\rangle$, and $\mathcal{Q}$ is given by

$$
\mathcal{Q}|\Psi\rangle=Q_{B}|\Psi\rangle+\left|\Psi_{0} * \Psi\right\rangle+\left|\Psi * \Psi_{0}\right\rangle
$$

The equation of motion at the tachyon vacuum,

$$
\mathcal{Q}|\Psi\rangle+|\Psi * \Psi\rangle=0
$$

can be solved by $\left|\Psi_{0}\right\rangle$ with an opposite sign,

$$
-\mathcal{Q}\left|\Psi_{0}\right\rangle+\left|\Psi_{0} * \Psi_{0}\right\rangle=0
$$

and it describes a D25-brane as an excitation from the tachyon vacuum.

It was conjectured in [41] that $\mathcal{Q}$ can be made purely of ghost fields by field redefinition, and string field theory with this conjectured form of the kinetic operator is called vacuum string field theory $[41,42,43]$. A more specific conjecture on $\mathcal{Q}$ was put forward later in [30]. The kinetic operator $\mathcal{Q}$ does not seem to be made purely of ghost fields when we expand the action around the approximate solution constructed by level truncation. It was conjectured [30] that there exists a one-parameter family of field redefinition which takes $\mathcal{Q}$ to the following form:

$$
\mathcal{Q}=\frac{Q}{\epsilon}[1+o(\epsilon)]
$$

where $Q$ is a $c$-ghost insertion at the open-string midpoint,

$$
Q=\frac{1}{2 i}(c(i)-c(-i))
$$

and $\epsilon$ corresponds to the parameter of the field redefinition. We denoted terms which vanishes in the limit $\epsilon \rightarrow 0$ by $o(\epsilon)$. In the singular limit $\epsilon \rightarrow 0$, the midpoint $c$-ghost insertion $Q$ dominates in the kinetic operator $\mathcal{Q}$ with an infinite coefficient.

Since the string field of vacuum string field theory is related to that of Witten's string field theory by field redefinition, the tachyon vacuum solution of Witten's string 
field theory with an opposite sign does not necessarily solve the equation of motion of vacuum string field theory. Interestingly, however, our leading-order solution with an opposite sign, $-\left|\Psi^{(0)}\right\rangle$, does solve the equation of motion of vacuum string field theory at the leading order without field redefinition.

Let us first compute the quantity $\left\langle\phi|Q| B_{t}(c)\right\rangle$. It is convenient to rewrite it as $\left\langle\phi|Q| B_{t}(c)\right\rangle=\left\langle B_{t}(c)|Q| \phi\right\rangle$. Since the operator $Q$ is mapped to

$$
\begin{aligned}
\frac{i}{2 p} \sqrt{1-t^{2}}[ & \left(1-i p \sqrt{1-t^{2}}\right)^{2} c\left(\frac{1}{1-i p \sqrt{1-t^{2}}}\right) \\
& \left.-\left(1+i p \sqrt{1-t^{2}}\right)^{2} c\left(\frac{1}{1+i p \sqrt{1-t^{2}}}\right)\right]
\end{aligned}
$$

by the conformal transformation $h_{t}(\xi)$, the quantity $\left\langle B_{t}(c)|Q| \phi\right\rangle$ is given by

$$
\begin{array}{r}
\left\langle B_{t}(c)|Q| \phi\right\rangle=\frac{i}{2 p^{2}} \sqrt{1-t^{2}}\left[\left(1-i p \sqrt{1-t^{2}}\right)^{2}\left\langle c(1) c\left(\frac{1}{1-i p \sqrt{1-t^{2}}}\right) h_{t} \circ \phi(0)\right\rangle\right. \\
\left.-\left(1+i p \sqrt{1-t^{2}}\right)^{2}\left\langle c(1) c\left(\frac{1}{1+i p \sqrt{1-t^{2}}}\right) h_{t} \circ \phi(0)\right\rangle\right] .
\end{array}
$$

In the limit $t \rightarrow 1$, the $c$ ghost coming from $Q$ approaches $c(1)$ so that the two operators can be replaced by the leading term of their OPE:

$$
c(1) c\left(\frac{1}{1 \pm i p \sqrt{1-t^{2}}}\right)=\mp i p \sqrt{2(1-t)} c \partial c(1)+O(1-t) .
$$

The leading term of $\left\langle B_{t}(c)|Q| \phi\right\rangle$ in the limit $t \rightarrow 1$ is given by

$$
\left\langle B_{t}(c)|Q| \phi\right\rangle=-\frac{2}{p}(1-t)\left\langle c \partial c(1) h_{B} \circ \phi(0)\right\rangle+O\left((1-t)^{2}\right) .
$$

Note that terms of $O\left((1-t)^{3 / 2}\right)$ cancel so that the next-to-leading order is $O\left((1-t)^{2}\right)$. Since the leading term is proportional to that of $\left\langle\phi \mid B_{t}(c) * B_{t}(c)\right\rangle$ in $(2.41)$, our solution with an opposite sign, $-\left|\Psi^{(0)}\right\rangle$, solves the equation of motion of vacuum string field theory up to $O(\sqrt{1-t})$,

$$
-\left\langle\phi|\mathcal{Q}| \Psi^{(0)}\right\rangle+\left\langle\phi \mid \Psi^{(0)} * \Psi^{(0)}\right\rangle=O(\sqrt{1-t})
$$

for any state $|\phi\rangle$ in the Fock space if the scaling between $\epsilon$ and $1-t$ is given by

$$
\epsilon=2(1-t)
$$

As we mentioned in the Introduction, it is easily understood why $\left|B_{t}(c)\right\rangle$ with an appropriate normalization solves the equation of motion of vacuum string field theory 
at the leading order. Both in $\left\langle\phi|Q| B_{t}(c)\right\rangle$ and in $\left\langle\phi \mid B_{t}(c) * B_{t}(c)\right\rangle$, the two $c$ ghosts approach the midpoint of the boundary of the surface and can be replaced by $c \partial c$ which is the leading term of the OPE. The existence of our approximate solution of Witten's string field theory seems to be consistent with the vacuum string field theory conjecture at this order. It would be interesting to explore the relation between our approach and vacuum string field theory in a more systematic way.

It was pointed out in [31] that subleading terms in (3.5) are necessary in order for vacuum string field theory to have a parameter corresponding to the string coupling constant. While the leading term $Q / \epsilon$ dominates in $\left\langle\phi_{1}|\mathcal{Q}| \phi_{2}\right\rangle$ for any pair of states $\left|\phi_{1}\right\rangle$ and $\left|\phi_{2}\right\rangle$ in the Fock space, the subleading terms may contribute at the same order as the leading term in other quantities. For example, $\left\langle\phi|Q| B_{t}(c)\right\rangle / \epsilon$ and $\left\langle\phi\left|Q_{B}\right| B_{t}(c)\right\rangle$ are the same order when $\epsilon \sim 1-t$. Therefore, the coefficient in (3.12) can be modified if we take into account the subleading terms.

Let us next consider the equation of motion of vacuum string field theory contracted with the solution itself. The computation of $\left\langle B_{t}(c)|Q| B_{t}(c)\right\rangle$ is almost parallel to that of $\left\langle B_{t}(c)\left|Q_{B}\right| B_{t}(c)\right\rangle$. The operator $Q$ is mapped to

$$
-\frac{1-t^{2}}{2\left(1+t^{2}\right)}(c(i)+c(-i))
$$

in the $z$ coordinate we introduced for the computation of $\left\langle B_{t}(c)\left|Q_{B}\right| B_{t}(c)\right\rangle$ so that the density of $\left\langle B_{t}(c)|Q| B_{t}(c)\right\rangle$ is given by

$$
\begin{aligned}
& \left\langle B_{t}(c)|Q| B_{t}(c)\right\rangle_{\text {density }} \\
& =-\frac{\left(1-t^{2}\right)^{2}}{8}\left[\langle c(-1) c(i) c(1)\rangle_{\text {density }}+\langle c(-1) c(-i) c(1)\rangle_{\text {density }}\right] \\
& =\left(1-t^{2}\right)^{2} .
\end{aligned}
$$

Therefore, the quantity $\left\langle\Psi^{(0)}|Q| \Psi^{(0)}\right\rangle / \epsilon$ has a finite density in the limit $t \rightarrow 1$ if $\epsilon$ scales as $1-t$. When $\epsilon=2(1-t)$, the ratio of $\left\langle\Psi^{(0)}|Q| \Psi^{(0)}\right\rangle / \epsilon$ to $\left\langle\Psi^{(0)} \mid \Psi^{(0)} * \Psi^{(0)}\right\rangle$ in the limit $t \rightarrow 1$ is given by

$$
\lim _{t \rightarrow 1} \frac{1}{2(1-t)} \frac{\left\langle\Psi^{(0)}|Q| \Psi^{(0)}\right\rangle}{\left\langle\Psi^{(0)} \mid \Psi^{(0)} * \Psi^{(0)}\right\rangle}=2^{\frac{13}{2}} \cdot 3^{-\frac{19}{4}} \simeq 0.4902 .
$$

The deviation of the value from 1 is much worse than the case of (2.52). In other words, the analogues of the conditions (i) and (ii-a) for vacuum string field theory are not compatible for the combination of $Q / \epsilon$ and $-\left|\Psi^{(0)}\right\rangle$ at the leading order. This result is independent of the scaling among $t, \epsilon$, and the normalization of the solution in (2.42). To make this point clearer, let us compute the following quantity introduced in $[31]$ :

$$
\frac{\langle\Psi * \Psi \mid \phi\rangle}{\langle\Psi|\mathcal{Q}| \phi\rangle} \frac{\langle\Psi|\mathcal{Q}| \Psi\rangle}{\langle\Psi * \Psi \mid \Psi\rangle}
$$


If this quantity is different from 1 , the equation of motion contracted with the solution itself is not compatible with the one contracted with a state $|\phi\rangle$ in the Fock space. This quantity is independent of the normalizations of $\mathcal{Q},|\Psi\rangle$, and $|\phi\rangle$ so that if $\mathcal{Q}$ is dominated by $Q / \epsilon$ and $|\Psi\rangle$ is dominated by $\left|\Psi^{(0)}\right\rangle$ in the limit $t \rightarrow 1$, it reduces to

$$
\lim _{t \rightarrow 1} \frac{\left\langle B_{t}(c) * B_{t}(c) \mid \phi\right\rangle}{\left\langle B_{t}(c)|Q| \phi\right\rangle} \frac{\left\langle B_{t}(c)|Q| B_{t}(c)\right\rangle}{\left\langle B_{t}(c) * B_{t}(c) \mid B_{t}(c)\right\rangle}=2^{\frac{13}{2}} \cdot 3^{-\frac{19}{4}} \simeq 0.4902 .
$$

A similar result was derived for the twisted regulated butterfly state $\left|B_{t}^{\prime}\right\rangle$ in [31]:

$$
\lim _{t \rightarrow 1} \frac{\left\langle B_{t}^{\prime} * B_{t}^{\prime} \mid \phi\right\rangle}{\left\langle B_{t}^{\prime}|Q| \phi\right\rangle} \frac{\left\langle B_{t}^{\prime}|Q| B_{t}^{\prime}\right\rangle}{\left\langle B_{t}^{\prime} * B_{t}^{\prime} \mid B_{t}^{\prime}\right\rangle}=\frac{\sqrt{2}}{3} \simeq 0.4714
$$

In both cases, the analogues of the conditions (i) and (ii-a) for vacuum string field theory are not compatible if we assume that $Q / \epsilon$ dominates in the kinetic operator and the solution is dominated by either $\left|B_{t}(c)\right\rangle$ or $\left|B_{t}^{\prime}\right\rangle$ with an appropriate normalization, and this conclusion holds whatever scaling we may take for $t, \epsilon$, and the normalization of the solution. As was demonstrated in [31], however, subleading terms of the kinetic operator $\mathcal{Q}$ can contribute to the quantity $\langle\Psi|\mathcal{Q}| \Psi\rangle$ at the same order as the leading term given by $Q / \epsilon$. Subleading terms of the solution may also contribute to $\langle\Psi|\mathcal{Q}| \Psi\rangle$ and $\langle\Psi * \Psi \mid \Psi\rangle$ at the same order. In fact, as we will show in the next section, the condition (ii-a) can be satisfied with better precision when we incorporate next-to-leading terms of the solution in the case of Witten's string field theory. Contributions from subleading terms in $\mathcal{Q}$ and in the solution may provide a resolution of the incompatibility in (3.17) or in (3.18). However, the relevance of the subleading terms in $\mathcal{Q}$ may ruin the factorization of the matter and ghost sectors at the leading term. We thus recognize that this is an important issue for the vacuum string field theory conjecture to be studied further.

\section{Solutions at the next-to-leading order}

\subsection{Solving the equation of motion at the next-to-leading or- der}

The solution $\left|\Psi^{(0)}\right\rangle$ in Section 2 solves the equation of motion of Witten's string field theory up to $O(\sqrt{1-t})$ :

$$
\left\langle\phi\left|Q_{B}\right| \Psi^{(0)}\right\rangle+\left\langle\phi \mid \Psi^{(0)} * \Psi^{(0)}\right\rangle=O(\sqrt{1-t}) .
$$

Let us try to improve the solution such that the equation is satisfied up to a higher power of $1-t$. 
There are two important sources of contributions at the next-to-leading order. First, there are contributions from the next-to-leading terms of the OPE in $\left\langle\phi \mid B_{t}(c) * B_{t}(c)\right\rangle$. Since the OPE of $c(-z)$ and $c(z)$ is given by

$$
c(-z) c(z)=2 z c \partial c(0)+\frac{1}{3} z^{3} c \partial^{3} c(0)-z^{3} \partial c \partial^{2} c(0)+O\left(z^{5}\right),
$$

the inner products $\left\langle\phi \mid B\left(c \partial^{3} c\right)\right\rangle$ and $\left\langle\phi \mid B\left(\partial c \partial^{2} c\right)\right\rangle$ will appear in $\left\langle\phi \mid B_{t}(c) * B_{t}(c)\right\rangle$ at the next-to-leading order.

Second, while the conformal transformations $f_{t}(\xi)$ and $\tilde{f}_{t}(\xi)$ coincide at the leading order, they differ at the next-to-leading order:

$$
\begin{aligned}
f_{t}(\xi) & =\frac{\xi}{\sqrt{1+t^{2} \xi^{2}}}=\frac{\xi}{\sqrt{1+\xi^{2}}}+(1-t)\left(\frac{\xi}{\sqrt{1+\xi^{2}}}\right)^{3}+O\left((1-t)^{2}\right) \\
& =f_{B}(\xi)+(1-t) f_{B}(\xi)^{3}+O\left((1-t)^{2}\right), \\
\tilde{f}_{t}(\xi) & =f_{B}(\xi)+\frac{1}{\sqrt{3}}(1-t) f_{B}(\xi)^{3}+O\left((1-t)^{2}\right) .
\end{aligned}
$$

The next-to-leading terms are proportional to $f_{B}(\xi)^{3}$ in both cases, but the coefficients are different. Up to this order, the conformal transformation $z=f_{t}(\xi)$ can be regarded as a combination of two transformations given by $\tilde{z}=f_{B}(\xi)$ and an infinitesimal one $z=\tilde{z}+(1-t) \tilde{z}^{3}+O\left((1-t)^{2}\right)$. Therefore, $f_{t} \circ \phi(0)$ can be represented in the expansion with respect to $1-t$ as follows:

$$
f_{t} \circ \phi(0)=f_{B} \circ \phi(0)+(1-t) \oint \frac{d z}{2 \pi i} z^{3} T(z) f_{B} \circ \phi(0)+O\left((1-t)^{2}\right),
$$

where the contour encircles the point $f_{B} \circ \phi(0)$ counterclockwise. Similarly, $\tilde{f}_{t} \circ \phi(0)$ is given by

$$
\widetilde{f}_{t} \circ \phi(0)=f_{B} \circ \phi(0)+\frac{1}{\sqrt{3}}(1-t) \oint \frac{d z}{2 \pi i} z^{3} T(z) f_{B} \circ \phi(0)+O\left((1-t)^{2}\right) .
$$

As we have shown in Section $2,\left\langle\phi\left|Q_{B}\right| B_{t}(c)\right\rangle$ is equal to $\left\langle\phi \mid B_{t}(c \partial c)\right\rangle$. Let us explicitly evaluate it up to the next-to-leading order in $1-t$. Since

$$
I \circ f_{t} \circ \phi(0)=I \circ f_{B} \circ \phi(0)-(1-t) \oint \frac{d z}{2 \pi i} \frac{1}{z} T(z) I \circ f_{B} \circ \phi(0)+O\left((1-t)^{2}\right),
$$

where the contour encircles the point $I \circ f_{B}(0)=\infty$ counterclockwise, the quantity $\left\langle\phi \mid B_{t}(c \partial c)\right\rangle$ is given by

$$
\begin{aligned}
\left\langle\phi \mid B_{t}(c \partial c)\right\rangle= & \left\langle I \circ f_{t} \circ \phi(0) c \partial c(0)\right\rangle \\
= & \left\langle I \circ f_{B} \circ \phi(0) c \partial c(0)\right\rangle+(1-t)\left\langle I \circ f_{B} \circ \phi(0) \oint \frac{d z}{2 \pi i} \frac{1}{z} T(z) c \partial c(0)\right\rangle \\
& +O\left((1-t)^{2}\right)
\end{aligned}
$$


where the contour encircles the origin counterclockwise. We have to compute the first regular term in the OPE of $T(z)$ and $c \partial c(0)$. The energy-momentum tensor $T(z)$ is given by

$$
T(z)=T^{m}(z)+:(\partial b) c:(z)-2 \partial(: b c:)(z),
$$

and its OPE with $c \partial c(w)$ is

$$
\begin{aligned}
T(z) c \partial c(w)= & -\frac{1}{(z-w)^{2}} c \partial c(w)+\frac{1}{z-w} c \partial^{2} c(w) \\
& +\frac{2}{3} c \partial^{3} c(w)-\frac{3}{2} \partial c \partial^{2} c(w)+c \partial c T^{m}(w)+O(z-w) .
\end{aligned}
$$

Therefore, $\left\langle\phi \mid B_{t}(c \partial c)\right\rangle$ is given by

$$
\begin{aligned}
\left\langle\phi \mid B_{t}(c \partial c)\right\rangle= & \langle\phi \mid B(c \partial c)\rangle \\
& +(1-t)\left[\frac{2}{3}\left\langle\phi \mid B\left(c \partial^{3} c\right)\right\rangle-\frac{3}{2}\left\langle\phi \mid B\left(\partial c \partial^{2} c\right)\right\rangle+\left\langle\phi \mid B\left(c \partial c T^{m}\right)\right\rangle\right] \\
& +O\left((1-t)^{2}\right) .
\end{aligned}
$$

To summarize, contributions from both sources consist of $\left\langle\phi \mid B\left(c \partial^{3} c\right)\right\rangle,\left\langle\phi \mid B\left(\partial c \partial^{2} c\right)\right\rangle$, and $\left\langle\phi \mid B\left(c \partial c T^{m}\right)\right\rangle$. The mass dimension of the operators inserted in these states is 1 , which is that of $c \partial c$ in the leading term plus 2. In addition, $\langle\phi \mid B(c \partial c)\rangle$ appearing at the leading order will also appear at the next-to-leading order. In order to satisfy the equation of motion up to the next-to-leading order, let us incorporate a set of states $\left|B_{t}(\mathcal{O})\right\rangle$ with the mass dimension of $\mathcal{O}$ being 1 into our ansatz. There are three such operators which consist of $b, c$, and $T^{m}$ and have the correct ghost number: $\left|B_{t}\left(\partial^{2} c\right)\right\rangle$, $\left|B_{t}\left(c T^{m}\right)\right\rangle$, and $\left|B_{t}(: b c \partial c:)\right\rangle$. Our ansatz then takes the following form:

$$
\begin{aligned}
\left|\Psi^{(2)}\right\rangle= & \frac{x}{\sqrt{1-t}}\left|B_{t}(c)\right\rangle \\
& +\sqrt{1-t}\left[u\left|B_{t}\left(\partial^{2} c\right)\right\rangle+v\left|B_{t}\left(c T^{m}\right)\right\rangle+w\left|B_{t}(: b c \partial c:)\right\rangle\right],
\end{aligned}
$$

where the $t$ dependence in the coefficients has been chosen for later convenience. The four operators $c, \partial^{2} c, c T^{m}$, and : $b c \partial c$ : correspond to $c_{1}|0\rangle, 2 c_{-1}|0\rangle, L_{-2}^{m} c_{1}|0\rangle$, and $-b_{-2} c_{0} c_{1}|0\rangle$, respectively, which constitute all the states up to level 2 in level truncation without gauge fixing.

Let us compute $\left\langle\phi\left|Q_{B}\right| \Psi^{(2)}\right\rangle$ and $\left\langle\phi \mid \Psi^{(2)} * \Psi^{(2)}\right\rangle$. Since

$$
\left\langle\phi\left|Q_{B}\right| B_{t}(\mathcal{O})\right\rangle=\left\langle\phi \mid B_{t}\left(Q_{B} \cdot \mathcal{O}\right)\right\rangle
$$

for any operator $\mathcal{O}$, we need to derive the BRST transformations of $\partial^{2} c, c T^{m}$, and $: b c \partial c$ : for the computation of $\left\langle\phi\left|Q_{B}\right| \Psi^{(2)}\right\rangle$. The BRST transformation of $\partial^{2} c$ can be easily derived from that of $c$ :

$$
Q_{B} \cdot \partial^{2} c(w)=\partial^{2}\left(Q_{B} \cdot c(w)\right)=\partial c \partial^{2} c(w)+c \partial^{3} c(w) .
$$


From the OPE's,

$$
\begin{aligned}
j_{B}(z) c T^{m}(w) \sim & -\frac{13}{(z-w)^{3}} c \partial c(w)-\frac{13}{2} \frac{1}{(z-w)^{2}} c \partial^{2} c(w) \\
& -\frac{13}{6} \frac{1}{z-w} c \partial^{3} c(w)-\frac{1}{z-w} c \partial c T^{m}(w), \\
j_{B}(z): b c \partial c:(w) \sim & \frac{6}{(z-w)^{3}} c \partial c(w)+\frac{3}{2} \frac{1}{(z-w)^{2}} c \partial^{2} c(w)+\frac{2}{3} \frac{1}{z-w} c \partial^{3} c(w) \\
& -\frac{3}{2} \frac{1}{z-w} \partial c \partial^{2} c(w)+\frac{1}{z-w} c \partial c T^{m}(w),
\end{aligned}
$$

the BRST transformations of $c T^{m}(w)$ and $: b c \partial c:(w)$ are given by

$$
\begin{aligned}
Q_{B} \cdot c T^{m}(w) & =-\frac{13}{6} c \partial^{3} c(w)-c \partial c T^{m}(w), \\
Q_{B} \cdot: b c \partial c:(w) & =\frac{2}{3} c \partial^{3} c(w)-\frac{3}{2} \partial c \partial^{2} c(w)+c \partial c T^{m}(w) .
\end{aligned}
$$

As a check, we can verify that $Q_{B} \cdot j_{B}(w)$ vanishes from these results. The quantity $\left\langle\phi\left|Q_{B}\right| \Psi^{(2)}\right\rangle$ is then given by

$$
\begin{aligned}
\left\langle\phi\left|Q_{B}\right| \Psi^{(2)}\right\rangle= & \frac{x}{\sqrt{1-t}}\left\langle\phi \mid B_{t}(c \partial c)\right\rangle+\sqrt{1-t}\left[\left(u-\frac{13}{6} v+\frac{2}{3} w\right)\left\langle\phi \mid B_{t}\left(c \partial^{3} c\right)\right\rangle\right. \\
& \left.+\left(u-\frac{3}{2} w\right)\left\langle\phi \mid B_{t}\left(\partial c \partial^{2} c\right)\right\rangle+(-v+w)\left\langle\phi \mid B_{t}\left(c \partial c T^{m}\right)\right\rangle\right] \\
= & \frac{x}{\sqrt{1-t}}\langle\phi \mid B(c \partial c)\rangle+\sqrt{1-t}\left[\left(\frac{2}{3} x+u-\frac{13}{6} v+\frac{2}{3} w\right)\left\langle\phi \mid B\left(c \partial^{3} c\right)\right\rangle\right. \\
& \left.+\left(-\frac{3}{2} x+u-\frac{3}{2} w\right)\left\langle\phi \mid B\left(\partial c \partial^{2} c\right)\right\rangle+(x-v+w)\left\langle\phi \mid B\left(c \partial c T^{m}\right)\right\rangle\right] \\
& +O\left((1-t)^{\frac{3}{2}}\right) .
\end{aligned}
$$

The computation of $\left\langle\phi \mid \Psi^{(2)} * \Psi^{(2)}\right\rangle$ takes the same steps as in the case of the leading order. First, we have to map the operators inserted at the origin in the coordinate $z^{\prime}=I \circ f_{t}(\xi)$ to $\pm 1 / \beta$ in the $z$ coordinate using the relation (2.18). The operator $\partial^{2} c$ is not a primary field, but its conformal transformation law can be easily derived from that of $c$. We will also need the transformation law of $\partial c$ later. They are given by

$$
\begin{aligned}
\partial c(f(z)) & \rightarrow \partial c(z)+\frac{f^{\prime \prime}(z)}{f^{\prime}(z)} c(z), \\
\partial^{2} c(f(z)) & \rightarrow \frac{1}{f^{\prime}(z)} \partial^{2} c(z)+\frac{f^{\prime \prime}(z)}{f^{\prime}(z)^{2}} \partial c(z)+\left\{\frac{f^{\prime \prime \prime}(z)}{f^{\prime}(z)^{2}}-\frac{f^{\prime \prime}(z)^{2}}{f^{\prime}(z)^{3}}\right\} c(z) .
\end{aligned}
$$

The conformal transformation of $c T^{m}$ can be obtained by evaluating the Schwarzian derivative with $c=26$ :

$$
c T^{m}(f(z)) \rightarrow \frac{1}{f^{\prime}(z)} c T^{m}(z)+\left\{-\frac{13}{6} \frac{f^{\prime \prime \prime}(z)}{f^{\prime}(z)^{2}}+\frac{13}{4} \frac{f^{\prime \prime}(z)^{2}}{f^{\prime}(z)^{3}}\right\} c(z) .
$$


The last operator : $b c \partial c$ : can be written as a linear combination of $\partial^{2} c, c T^{m}$, and $j_{B}$. We have obtained the conformal transformations of $\partial^{2} c$ and $c T^{m}$, and the BRST current $j_{B}$ is a primary field:

$$
j_{B}(f(z)) \rightarrow \frac{1}{f^{\prime}(z)} j_{B}(z)
$$

Therefore, the transformation law of $: b c \partial c$ : is given by

$$
: b c \partial c:(f(z)) \rightarrow \frac{1}{f^{\prime}(z)}: b c \partial c:(z)-\frac{3}{2} \frac{f^{\prime \prime}(z)}{f^{\prime}(z)^{2}} \partial c(z)+\left\{\frac{2}{3} \frac{f^{\prime \prime \prime}(z)}{f^{\prime}(z)^{2}}-\frac{7}{4} \frac{f^{\prime \prime}(z)^{2}}{f^{\prime}(z)^{3}}\right\} c(z) .
$$

The derivatives $d^{2} z^{\prime} / d z^{2}$ and $d^{3} z^{\prime} / d z^{3}$ at $z= \pm 1 / \beta$ can be computed from (2.18) as follows:

$$
\begin{aligned}
& \left.\frac{d^{2} z^{\prime}}{d z^{2}}\right|_{z= \pm \frac{1}{\beta}}= \pm \frac{\beta^{2} d^{2}\left(3 \beta^{2}-7 d^{2}\right) \sqrt{1-t^{4}}}{2 \sqrt{3}\left(\beta^{2}+d^{2}\right)^{2}} \\
& \left.\frac{d^{3} z^{\prime}}{d z^{3}}\right|_{z= \pm \frac{1}{\beta}}=\frac{\beta^{3} d^{4}\left(122 d^{2}+27 d^{2} t^{2}-120 \beta^{2}\right) \sqrt{1-t^{4}}}{8 \sqrt{3}\left(\beta^{2}+d^{2}\right)^{3}} .
\end{aligned}
$$

The conformal transformations of $\partial^{2} c, c T^{m}$, and : bc $\partial c$ : can be calculated by plugging these derivatives into their transformation laws.

In Subsection 2.2, we made the conformal map $z /(z+p)$ to bring the infinity to a finite point in studying the limit $t \rightarrow 1$. This is convenient when we explicitly compute the inner product $\left\langle\phi \mid \Psi^{(2)} * \Psi^{(2)}\right\rangle$ for a given $|\phi\rangle$ because the operator $\phi(0)$ in the original coordinate is also mapped to a finite point. In solving the equation of motion, however, it is sufficient to make a simple inversion which brings the operators at $z= \pm 1 / \beta$ to $\mp \beta$. After the inversion, the operators at $z^{\prime}=0$ are mapped as follows:

$$
\begin{aligned}
& \frac{x}{\sqrt{1-t}} c(0)+\sqrt{1-t}\left[u \partial^{2} c(0)+v c T^{m}(0)+w: b c \partial c:(0)\right] \\
& \rightarrow \frac{1}{\beta}\left(\frac{2}{\sqrt{3}} x+\frac{55}{27 \sqrt{3}} u-\frac{247}{72 \sqrt{3}} v+\frac{\sqrt{3}}{8} w+O(1-t)\right) c(\mp \beta) \\
& \quad \mp\left(-\frac{7}{6 \sqrt{3}} u+\frac{7}{4 \sqrt{3}} w+O(1-t)\right) \partial c(\mp \beta) \\
& \quad+\beta\left(\frac{\sqrt{3}}{2}+O(1-t)\right)\left[u \partial^{2} c(\mp \beta)+v c T^{m}(\mp \beta)+w: b c \partial c:(\mp \beta)\right] .
\end{aligned}
$$

We have only presented the leading terms in the limit $t \rightarrow 1$ here, but the expression for any $t$ is also available. Since $\beta$ goes to zero as $t \rightarrow 1$, the operators at $\mp \beta$ can be 
expanded in terms of operators at the origin. If we define $V_{ \pm}(z)$ by

$$
V_{ \pm}(z) \equiv\left(\begin{array}{c}
\beta^{-1} c(z) \\
\pm \partial c(z) \\
\beta \partial^{2} c(z) \\
\beta c T^{m}(z) \\
\beta: b c \partial c:(z)
\end{array}\right),
$$

relevant OPE's can be expressed and evaluated as follows:

$$
\begin{aligned}
& \frac{1}{2} V_{-}(-\beta) V_{+}^{T}(\beta)-\frac{1}{2} V_{+}(\beta) V_{-}^{T}(-\beta) \\
& =\frac{1}{\beta} \mathcal{M}_{1} c \partial c(0)+\beta \mathcal{M}_{2} c \partial^{3} c(0)+\beta \mathcal{M}_{3} \partial c \partial^{2} c(0)+\beta \mathcal{M}_{4} c \partial c T^{m}(0) \\
& \quad+O\left(\beta^{3}\right)
\end{aligned}
$$

where $V_{ \pm}^{T}(z)$ denotes the transpose of $V_{ \pm}(z)$ and

$$
\begin{aligned}
& \mathcal{M}_{1}=\left(\begin{array}{ccccc}
2 & 1 & 0 & 0 & -\frac{1}{2} \\
1 & 0 & 0 & 0 & \frac{1}{4} \\
0 & 0 & 0 & 0 & -\frac{1}{4} \\
0 & 0 & 0 & \frac{13}{8} & 0 \\
-\frac{1}{2} & \frac{1}{4} & -\frac{1}{4} & 0 & -\frac{3}{8}
\end{array}\right), \quad \mathcal{M}_{2}=\left(\begin{array}{ccccc}
\frac{1}{3} & \frac{1}{2} & 1 & 0 & -\frac{1}{4} \\
\frac{1}{2} & 0 & 0 & 0 & \frac{1}{8} \\
1 & 0 & 0 & 0 & -\frac{1}{8} \\
0 & 0 & 0 & \frac{13}{48} & 0 \\
-\frac{1}{4} & \frac{1}{8} & -\frac{1}{8} & 0 & -\frac{7}{48}
\end{array}\right), \\
& \mathcal{M}_{3}=\left(\begin{array}{ccccc}
-1 & -\frac{3}{2} & -1 & 0 & -\frac{1}{4} \\
-\frac{3}{2} & -2 & -1 & 0 & \frac{1}{8} \\
-1 & -1 & 0 & 0 & -\frac{1}{8} \\
0 & 0 & 0 & -\frac{13}{16} & 0 \\
-\frac{1}{4} & \frac{1}{8} & -\frac{1}{8} & 0 & \frac{15}{16}
\end{array}\right), \quad \mathcal{M}_{4}=\left(\begin{array}{ccccc}
0 & 0 & 0 & 2 & 0 \\
0 & 0 & 0 & 1 & 0 \\
0 & 0 & 0 & 0 & 0 \\
2 & 1 & 0 & 1 & -\frac{1}{2} \\
0 & 0 & 0 & -\frac{1}{2} & 0
\end{array}\right) .
\end{aligned}
$$

Therefore, the quantity $\left\langle\phi \mid \Psi^{(2)} * \Psi^{(2)}\right\rangle$ can be expanded as follows:

$$
\begin{aligned}
\left\langle\phi \mid \Psi^{(2)} * \Psi^{(2)}\right\rangle= & \frac{\mathcal{F}_{1}}{\sqrt{1-t}}\left\langle\phi \mid \widetilde{B}_{t}(c \partial c)\right\rangle+\sqrt{1-t}\left[\mathcal{F}_{2}\left\langle\phi \mid \widetilde{B}_{t}\left(c \partial^{3} c\right)\right\rangle+\mathcal{F}_{3}\left\langle\phi \mid \widetilde{B}_{t}\left(\partial c \partial^{2} c\right)\right\rangle\right. \\
& \left.+\mathcal{F}_{4}\left\langle\phi \mid \widetilde{B}_{t}\left(c \partial c T^{m}\right)\right\rangle\right]+O\left((1-t)^{\frac{3}{2}}\right),
\end{aligned}
$$

where $\mathcal{F}_{1}, \mathcal{F}_{2}, \mathcal{F}_{3}$, and $\mathcal{F}_{4}$ are functions of $x, u, v$, and $w$, and we have defined

$$
\left\langle\phi \mid \widetilde{B}_{t}(\mathcal{O})\right\rangle=\left\langle I \circ \widetilde{f}_{t} \circ \phi(0) \mathcal{O}(0)\right\rangle
$$

for any state $|\phi\rangle$ in the Fock space. From (4.26) and (4.28), the functions $\mathcal{F}_{1}, \mathcal{F}_{2}, \mathcal{F}_{3}$, and $\mathcal{F}_{4}$ can be computed. For example, $\mathcal{F}_{1}$ is given by

$$
\mathcal{F}_{1}=\frac{3^{\frac{3}{4}}}{\sqrt{2}}\left(\frac{8 x^{2}}{3}+\frac{314 x u}{81}+\frac{2585 u^{2}}{2187}-\frac{247 x v}{27}-\frac{38779 u v}{5832}+\frac{35243 v^{2}}{3888}\right.
$$




$$
\left.+\frac{7 x w}{3}+\frac{919 u w}{648}-\frac{1729 v w}{432}+\frac{w^{2}}{2}\right)+O(1-t)
$$

The leading part of the equation $\left\langle\phi\left|Q_{B}\right| \Psi^{(2)}\right\rangle+\left\langle\phi \mid \Psi^{(2)} * \Psi^{(2)}\right\rangle=0$, which is proportional to $\langle\phi \mid B(c \partial c)\rangle$ and is of $O(1 / \sqrt{1-t})$, is therefore given by

$$
\begin{aligned}
x+\frac{3^{\frac{3}{4}}}{\sqrt{2}}\left(\frac{8 x^{2}}{3}+\frac{314 x u}{81}+\right. & \frac{2585 u^{2}}{2187}-\frac{247 x v}{27}-\frac{38779 u v}{5832}+\frac{35243 v^{2}}{3888} \\
& \left.+\frac{7 x w}{3}+\frac{919 u w}{648}-\frac{1729 v w}{432}+\frac{w^{2}}{2}\right)=0 .
\end{aligned}
$$

As we mentioned, there are two important sources of terms at the next-to-leading order. The first kind of terms come from $\mathcal{F}_{2}, \mathcal{F}_{3}$, and $\mathcal{F}_{4}$. The second kind of terms which come from the difference between $f_{t}(\xi)$ and $\widetilde{f}_{t}(\xi)$ at $O(1-t)$ can be taken into account by replacing $x$ with $(1-1 / \sqrt{3}) x$ in the coefficients in front of $\left\langle\phi \mid B\left(c \partial^{3} c\right)\right\rangle,\left\langle\phi \mid B\left(\partial c \partial^{2} c\right)\right\rangle$, and $\left\langle\phi \mid B\left(c \partial c T^{m}\right)\right\rangle$ in (4.18) because of the equation (4.33). By computing $\mathcal{F}_{2}, \mathcal{F}_{3}$, and $\mathcal{F}_{4}$ from $(4.26)$ and $(4.28)$, we obtain the following three equations at the next-toleading order:

$$
\begin{aligned}
& \frac{2}{3}\left(1-\frac{1}{\sqrt{3}}\right) x+u-\frac{13 v}{6}+\frac{2 w}{3}+\frac{\sqrt{2}}{3^{\frac{3}{4}}}\left(\frac{4 x^{2}}{9}+\frac{517 x u}{243}+\frac{22385 u^{2}}{13122}-\frac{247 x v}{162}\right. \\
& \left.-\frac{127699 u v}{34992}+\frac{35243 v^{2}}{23328}+\frac{5 x w}{6}+\frac{965 u w}{1296}-\frac{1235 v w}{864}+\frac{w^{2}}{4}\right)=0 \\
& -\frac{3}{2}\left(1-\frac{1}{\sqrt{3}}\right) x+u-\frac{3 w}{2}+\frac{\sqrt{2}}{3^{\frac{3}{4}}}\left(-\frac{4 x^{2}}{3}-\frac{193 x u}{81}-\frac{3431 u^{2}}{4374}+\frac{247 x v}{54}\right. \\
& \left.+\frac{47671 u v}{11664}-\frac{35243 v^{2}}{7776}-\frac{9 x w}{2}-\frac{559 u w}{144}+\frac{247 v w}{32}-\frac{23 w^{2}}{12}\right)=0 \\
& \left(1-\frac{1}{\sqrt{3}}\right) x-v+w+\frac{\sqrt{2}}{3^{\frac{3}{4}}}\left(4 x v+\frac{157 u v}{54}-\frac{55 v^{2}}{9}+\frac{7 v w}{4}\right)=0
\end{aligned}
$$

There are also terms of $O(\sqrt{1-t})$ which are proportional to $\langle\phi \mid B(c \partial c)\rangle$. The equation coming from $\langle\phi \mid B(c \partial c)\rangle$ at this subleading order can be easily satisfied by introducing a subleading part of $x$. We will not compute it because, as we will see, it does not contribute to the energy density of the solution in the limit $t \rightarrow 1$.

We have the four equations (4.33), (4.34), (4.35), and (4.36) to solve for the four variables $x, u, v$, and $w$. We could not solve them analytically, but we found six nontrivial, real-valued solutions numerically by using Mathematica. They are given in Table 2. The numerical value of $x$ at the leading-order solution is

$$
x_{\text {leading }}=-\frac{3^{\frac{1}{4}}}{4 \sqrt{2}} \simeq-0.232651 .
$$


Table 2: Solutions at the next-to-leading order.

\begin{tabular}{|c|c|c|c|}
\hline$x$ & $u$ & $v$ & $w$ \\
\hline-3.94824 & 28.5424 & 15.5319 & 15.9191 \\
\hline 8.23295 & -10.1642 & -0.83021 & 0.450412 \\
\hline-0.170713 & 1.19342 & 0.726956 & 0.863933 \\
\hline-0.241241 & -0.486967 & -0.341072 & -0.479342 \\
\hline-0.329909 & 0.00107446 & 0.00924674 & 0.155003 \\
\hline-0.0000505996 & 0.0138804 & 0.00924674 & 0.00926889 \\
\hline
\end{tabular}

The value of $x$ in the fourth solution is fairly close to this value, and those of the third and fifth ones are not too far away. At this point, however, there are no proper reasons to select a subset among the six. In the next subsection, we will evaluate the energy density for these solutions, and it turns out that the fourth and fifth solutions are selected from the condition (ii-a) in the Introduction.

\subsection{Energy density at the next-to-leading order}

As we did for the leading-order solution $\left|\Psi^{(0)}\right\rangle$ in Subsection 2.3, let us compute $\left\langle\Psi^{(2)}\left|Q_{B}\right| \Psi^{(2)}\right\rangle$ and $\left\langle\Psi^{(2)} \mid \Psi^{(2)} * \Psi^{(2)}\right\rangle$. It will turn out to be convenient to introduce the following parametrization of $\left|\Psi^{(2)}\right\rangle$ for the computations of these quantities:

$$
\begin{aligned}
\left|\Psi^{(2)}\right\rangle= & \frac{\tilde{x}}{\sqrt{1-t^{4}}}\left|B_{t}(c)\right\rangle \\
& +\sqrt{1-t^{4}}\left[\tilde{u}\left|B_{t}\left(\partial^{2} c\right)\right\rangle+\tilde{v}\left|B_{t}\left(c T^{m}\right)\right\rangle+\tilde{w}\left|B_{t}(: b c \partial c:)\right\rangle\right] .
\end{aligned}
$$

Let us start with $\left\langle\Psi^{(2)}\left|Q_{B}\right| \Psi^{(2)}\right\rangle$. From the relation (2.45), the derivatives $d^{2} z^{\prime} / d z^{2}$ and $d^{3} z^{\prime} / d z^{3}$ at $z= \pm 1$ can be computed as follows:

$$
\left.\frac{d^{2} z^{\prime}}{d z^{2}}\right|_{z= \pm 1}=\mp \frac{\sqrt{1-t^{4}}}{2},\left.\quad \frac{d^{3} z^{\prime}}{d z^{3}}\right|_{z= \pm 1}=\frac{3\left(2+t^{2}\right) \sqrt{1-t^{4}}}{8} .
$$

The operators inserted at $z^{\prime}=0$ are mapped to $z=-1$ in the $z$ coordinate as

$$
\begin{aligned}
& \frac{\tilde{x}}{\sqrt{1-t^{4}}} c(0)+\sqrt{1-t^{4}}\left\{\tilde{u} \partial^{2} c(0)+\tilde{v} c T^{m}(0)+\tilde{w}: b c \partial c:(0)\right\} \\
\rightarrow & \left(\frac{\tilde{x}}{2}+\frac{2+3 t^{2}}{2} \tilde{u}-\frac{13 t^{2}}{4} \tilde{v}+\frac{2 t^{2}-3}{2} \tilde{w}\right) c(-1)+(2 \tilde{u}-3 \tilde{w}) \partial c(-1) \\
& +2 \tilde{u} \partial^{2} c(-1)+2 \tilde{v} c T^{m}(-1)+2 \tilde{w}: b c \partial c:(-1) .
\end{aligned}
$$


The BRST transformations of the operators at $z^{\prime}=0$ are mapped to $z=1$ in the $z$ coordinate as

$$
\begin{aligned}
& Q_{B} \cdot\left[\frac{\tilde{x}}{\sqrt{1-t^{4}}} c(0)+\sqrt{1-t^{4}}\left\{\tilde{u} \partial^{2} c(0)+\tilde{v} c T^{m}(0)+\tilde{w}: b c \partial c:(0)\right\}\right] \\
= & \left(\frac{\tilde{x}}{2}+\frac{2+3 t^{2}}{2} \tilde{u}-\frac{13 t^{2}}{4} \tilde{v}+\frac{2 t^{2}-3}{2} \tilde{w}\right) c \partial c(1)+(-2 \tilde{u}+3 \tilde{w}) c \partial^{2} c(1) \\
& +\left(2 \tilde{u}-\frac{13}{3} \tilde{v}+\frac{4}{3} \tilde{w}\right) c \partial^{3} c(1)+(2 \tilde{u}-3 \tilde{w}) \partial c \partial^{2} c(1)+(-2 \tilde{v}+2 \tilde{w}) c \partial c T^{m}(1) .
\end{aligned}
$$

It is tedious but straightforward to compute the correlation functions of these operators. The final result for $\left\langle\Psi^{(2)}\left|Q_{B}\right| \Psi^{(2)}\right\rangle$ is given by

$$
\begin{aligned}
\left\langle\Psi^{(2)}\left|Q_{B}\right| \Psi^{(2)}\right\rangle_{\text {density }}= & -\tilde{x}^{2}-6 t^{2} \tilde{x} \tilde{u}-\left(4+9 t^{4}\right) \tilde{u}^{2}+13 t^{2} \tilde{x} \tilde{v}+39 t^{4} \tilde{u} \tilde{v} \\
& +\left(13-\frac{169 t^{4}}{4}\right) \tilde{v}^{2}-4 t^{2} \tilde{x} \tilde{w}+12\left(1-t^{4}\right) \tilde{u} \tilde{w} \\
& -26\left(1-t^{4}\right) \tilde{v} \tilde{w}+4\left(1-t^{4}\right) \tilde{w}^{2} .
\end{aligned}
$$

We are interested in the limit $t \rightarrow 1$. As can be seen from this expression, the quantity $\left\langle\Psi^{(2)}\left|Q_{B}\right| \Psi^{(2)}\right\rangle$ has a finite limit as $t$ goes to 1 . Since

$$
\lim _{t \rightarrow 1} \tilde{x}=2 x, \quad \lim _{t \rightarrow 1} \tilde{u}=\frac{1}{2} u, \quad \lim _{t \rightarrow 1} \tilde{v}=\frac{1}{2} v, \quad \lim _{t \rightarrow 1} \tilde{w}=\frac{1}{2} w,
$$

it is given as follows:

$$
\begin{aligned}
& \lim _{t \rightarrow 1}\left\langle\Psi^{(2)}\left|Q_{B}\right| \Psi^{(2)}\right\rangle_{\text {density }} \\
& =-4 x^{2}-6 x u-\frac{13 u^{2}}{4}+13 x v+\frac{39 u v}{4}-\frac{117 v^{2}}{16}-4 x w .
\end{aligned}
$$

The computation of $\left\langle\Psi^{(2)} \mid \Psi^{(2)} * \Psi^{(2)}\right\rangle$ can be done in a similar way. From the relation (2.47), the derivatives $d^{2} z^{\prime} / d z^{2}$ and $d^{3} z^{\prime} / d z^{3}$ at $z=0$ and $z= \pm \sqrt{3}$ can be computed as follows:

$$
\begin{aligned}
& \left.\frac{d^{2} z^{\prime}}{d z^{2}}\right|_{z=0}=0,\left.\quad \frac{d^{3} z^{\prime}}{d z^{3}}\right|_{z=0}=\frac{3\left(10+27 t^{2}\right) \sqrt{1-t^{4}}}{8} \\
& \left.\frac{d^{2} z^{\prime}}{d z^{2}}\right|_{z= \pm \sqrt{3}}=\mp \frac{3 \sqrt{3\left(1-t^{4}\right)}}{16},\left.\quad \frac{d^{3} z^{\prime}}{d z^{3}}\right|_{z= \pm \sqrt{3}}=\frac{3\left(82+27 t^{2}\right) \sqrt{1-t^{4}}}{512} .
\end{aligned}
$$

The operators inserted at $z^{\prime}=0$ are mapped to $z=0$ and $z= \pm \sqrt{3}$ in the $z$ coordinate as

$$
\frac{\tilde{x}}{\sqrt{1-t^{4}}} c(0)+\sqrt{1-t^{4}}\left\{\tilde{u} \partial^{2} c(0)+\tilde{v} c T^{m}(0)+\tilde{w}: b c \partial c:(0)\right\}
$$




$$
\begin{aligned}
\rightarrow & \left(\frac{3}{2} \tilde{x}+\frac{10+27 t^{2}}{6} \tilde{u}-\frac{13\left(10+27 t^{2}\right)}{36} \tilde{v}+\frac{10+27 t^{2}}{9} \tilde{w}\right) c(0) \\
& +\frac{2}{3} \tilde{u} \partial^{2} c(0)+\frac{2}{3} \tilde{v} c T^{m}(0)+\frac{2}{3} \tilde{w}: b c \partial c:(0) \\
& \left(\frac{3}{8} \tilde{x}+\frac{34+27 t^{2}}{24} \tilde{u}-\frac{13\left(10+27 t^{2}\right)}{144} \tilde{v}-\frac{44-27 t^{2}}{36} \tilde{w}\right) c( \pm \sqrt{3}) \\
& \pm\left(-\frac{4 \sqrt{3}}{3} \tilde{u}+2 \sqrt{3} \tilde{w}\right) \partial c( \pm \sqrt{3}) \\
& +\frac{8}{3} \tilde{u} \partial^{2} c( \pm \sqrt{3})+\frac{8}{3} \tilde{v} c T^{m}( \pm \sqrt{3})+\frac{8}{3} \tilde{w}: b c \partial c:( \pm \sqrt{3})
\end{aligned}
$$

It is again tedious but straightforward to compute the correlation functions of these operators. The final expression of $\left\langle\Psi^{(2)} \mid \Psi^{(2)} * \Psi^{(2)}\right\rangle$ for an arbitrary $t$ is given in Appendix B. As in the case of $\left\langle\Psi^{(2)}\left|Q_{B}\right| \Psi^{(2)}\right\rangle$, it also has a finite limit as $t$ goes to 1 , which is given by

$$
\begin{aligned}
\lim _{t \rightarrow 1}\left\langle\Psi^{(2)} \mid \Psi^{(2)} * \Psi^{(2)}\right\rangle_{\text {density }}=-\frac{81 \sqrt{3} x^{3}}{8}-\frac{927 \sqrt{3} x^{2} u}{32}-\frac{3451 \sqrt{3} x u^{2}}{128} \\
-\frac{4205 \sqrt{3} u^{3}}{512}+\frac{4329 \sqrt{3} x^{2} v}{64}+\frac{49543 x u v}{128 \sqrt{3}}+\frac{1659931 u^{2} v}{9216 \sqrt{3}}-\frac{244673 x v^{2}}{512 \sqrt{3}} \\
-\frac{25201319 u v^{2}}{55296 \sqrt{3}}+\frac{43213963 v^{3}}{110592 \sqrt{3}}-\frac{315 \sqrt{3} x^{2} w}{16}-\frac{1095 \sqrt{3} x u w}{32} \\
-\frac{286195 u^{2} w}{6912 \sqrt{3}}+\frac{16835 x v}{64 \sqrt{3}}+\frac{175565 u v w}{768 \sqrt{3}}-\frac{8563555 v^{2} w}{27648 \sqrt{3}} \\
-\frac{403 \sqrt{3} x w^{2}}{32}-\frac{104687 u w^{2}}{3456 \sqrt{3}}+\frac{193843 v w^{2}}{2304 \sqrt{3}}-\frac{169 \sqrt{3} w^{3}}{64}
\end{aligned}
$$

We can now evaluate $\mathcal{R}\left[\Psi^{(2)}\right]$ and $\mathcal{E}\left[\Psi^{(2)}\right] / T_{25}$ in the limit $t \rightarrow 1$ for the numerical solutions we found in the previous subsection. The result is summarized in Table 3. The values of $\mathcal{R}$ for the fourth and fifth solutions are very close to -1 . Therefore, these solutions approximately satisfy the equation of motion even when it is contracted with the solution itself. Furthermore, the values of $\mathcal{E} / T_{25}$ for these two solutions are fairly close to -1 . They give approximately $109 \%$ and $88 \%$, respectively, of the D25-brane tension. Compared with the solution at the leading order which gives approximately $68 \%$ of the D25-brane tension, these values of the energy density for these solutions at the next-to-leading order are closer to the predicted value $-T_{25}$.

It is not surprising that we have found more than one numerical solution at the next-to-leading order because we do not fix gauge. Multiple numerical solutions were also found in the level truncation analysis without gauge fixing by Ellwood and Taylor [40], and there are two solutions at level 2. In fact, the values of the energy density 
Table 3: $\mathcal{R}$ and $\mathcal{E} / T_{25}$ in the limit $t \rightarrow 1$.

\begin{tabular}{|c||c||c|c|c|c|}
\hline $\mathcal{R}$ & $\mathcal{E} / T_{25}$ & $x$ & $u$ & $v$ & $w$ \\
\hline 0.143959 & -1188.83 & -3.95 & 28.5 & 15.5 & 15.9 \\
\hline-0.61934 & 98.9891 & 8.23 & -10.2 & -0.830 & 0.450 \\
\hline-0.822535 & 0.0897315 & -0.171 & 1.19 & 0.727 & 0.864 \\
\hline-0.998109 & -1.08981 & -0.241 & -0.487 & -0.341 & -0.479 \\
\hline-0.998909 & -0.882631 & -0.330 & 0.00107 & 0.00925 & 0.155 \\
\hline-0.326083 & $3.05927 \times 10^{-8}$ & -0.0000506 & 0.0139 & 0.00925 & 0.00927 \\
\hline
\end{tabular}

for our solutions are interestingly close to those for the two solutions at level 2 in level truncation. We will review the results of level truncation in the next subsection, and compare them with ours.

\subsection{Comparison with solutions by a variational method}

A state in the form of $\left|B_{t}(\mathcal{O})\right\rangle$ reduces to a state $|\mathcal{O}\rangle$ in the Fock space in the limit $t \rightarrow$ 0 . Therefore, the results of the ordinary level-truncation analysis can be reproduced simply by taking the limit $t \rightarrow 0$ of ours. In this limit, the parameters $\tilde{x}, \tilde{u}, \tilde{v}$, and $\tilde{w}$ reduce to $x, u, v$, and $w$, respectively, so that $\mathcal{E} / T_{25}$ in the limit is given by

$$
\begin{aligned}
& \left.\frac{\mathcal{E}}{T_{25}}\right|_{t=0}=2 \pi^{2}\left(-\frac{x^{2}}{2}-2 u^{2}+\frac{13 v^{2}}{2}+6 u w-13 v w+2 w^{2}\right. \\
& \quad-\frac{27 \sqrt{3} x^{3}}{64}-\frac{33 \sqrt{3} x^{2} u}{32}-\frac{19 x u^{2}}{16 \sqrt{3}}-\frac{u^{3}}{8 \sqrt{3}}+\frac{195 \sqrt{3} x^{2} v}{64}+\frac{715 x u v}{48 \sqrt{3}} \\
& \quad+\frac{1235 u^{2} v}{432 \sqrt{3}}-\frac{7553 x v^{2}}{192 \sqrt{3}}-\frac{83083 u v^{2}}{2592 \sqrt{3}}+\frac{272363 v^{3}}{5184 \sqrt{3}}-\frac{3 \sqrt{3} x^{2} w}{4}+\sqrt{3} x u w \\
& \left.+\frac{703 u^{2} w}{81 \sqrt{3}}+\frac{65 x v w}{6 \sqrt{3}}-\frac{65 u v w}{9 \sqrt{3}}-\frac{7553 v^{2} w}{324 \sqrt{3}}-\frac{x w^{2}}{\sqrt{3}}+\frac{94 u w^{2}}{81 \sqrt{3}}+\frac{65 v w^{2}}{27 \sqrt{3}}\right) .
\end{aligned}
$$

In the level-truncation analysis by Sen and Zwiebach [1], the Siegel gauge condition was imposed. It corresponds to setting the parameter $w$ to zero in our expression. The expression in [1] at level 2 is precisely reproduced by the following identification of the parameters:

$$
x_{\text {ours }}=-t_{S Z}, \quad u_{\text {ours }}=-\frac{1}{2} u_{S Z}, \quad v_{\text {ours }}=-\frac{1}{\sqrt{13}} v_{S Z}, \quad w_{\text {ours }}=0 .
$$


By taking variations with respect to $x, u$, and $v$, we numerically find the following stationary point:

$$
x_{c} \simeq-0.544204, \quad u_{c} \simeq-0.0950952, \quad v_{c} \simeq-0.0559637,
$$

which reproduces the result by Sen and Zwiebach at level 2 in the Siegel gauge:

$$
\frac{\mathcal{E}}{T_{25}} \simeq-0.959377
$$

The level-truncation analysis can be done without imposing a gauge condition, and it was studied by Rastelli and Zwiebach [39] and by Ellwood and Taylor [40]. By taking variations with respect to all of $x, u, v$, and $w$, we numerically find eight stationary points, which we present in Table 4 together with the values of $\mathcal{E} / T_{25}$. The values of $\mathcal{E} / T_{25}$ for the fifth and sixth solutions are relatively close to -1 , giving approximately $108 \%$ and $88 \%$ of the D25-brane tension, respectively. They are the two solutions found by Ellwood and Taylor in [40]. ${ }^{5}$ These values of the energy density are interestingly close to those of our solutions at the next-to-leading order. In the ordinary leveltruncation analysis without gauge fixing, there are no proper reasons to select the two solutions among the eight. In our case, we emphasize that we chose the two solutions in Subsection 4.2 by the condition (ii-a), not by the criterion that they give a better value for the energy density.

Table 4: Level truncation at level 2 without gauge fixing.

\begin{tabular}{|c||c|c|c|c|}
\hline $\mathcal{E} / T_{25}$ & $x$ & $u$ & $v$ & $w$ \\
\hline-64.2501 & -1.84905 & 9.8938 & 5.12224 & 5.24925 \\
\hline-103.454 & 1.83562 & -3.53656 & -1.04338 & -0.455299 \\
\hline 0.212599 & -0.212556 & 0.732749 & 0.440045 & 0.542256 \\
\hline 1303.36 & 0.551333 & -0.63015 & -1.4288 & -14.0212 \\
\hline-1.0778 & -0.590367 & -0.283678 & -0.191173 & -0.268326 \\
\hline-0.88015 & -0.572474 & 0.053691 & 0.0422479 & 0.180675 \\
\hline $8.16109 \times 10^{-11}$ & 0. & $1.19631 \times 10^{-6}$ & 0 & $7.97539 \times 10^{-7}$ \\
\hline $8.16104 \times 10^{-11}$ & 0. & $-1.19631 \times 10^{-6}$ & 0 & $-7.97538 \times 10^{-7}$ \\
\hline
\end{tabular}

We can also perform a similar variational analysis based on the expression for the energy density in the limit $t \rightarrow 1$. We numerically found six stationary points listed in Table 5 together with the values of $\mathcal{E} / T_{25}$ for those solutions. As can be seen from

\footnotetext{
${ }^{5}$ One of them with $88 \%$ of the D25-brane tension was first found by Rastelli and Zwiebach [39].
} 
the table, the values of $\mathcal{E} / T_{25}$ are precisely the same up to the order we computed as those for the first six solutions in Table 4. This seems to indicate that the set of configurations in the limit $t \rightarrow 1$ can be obtained from that of $t=0$ by field redefinition. Note, however, that our solutions at the next-to-leading order in Subsection 4.2 cannot be obtained simply by field redefinition from the solutions of level truncation at level 2 , which can be seen from the fact that the values of the energy density are close, but not exactly the same.

Table 5: Variational analysis based on the expression in the limit $t \rightarrow 1$.

\begin{tabular}{|c||c|c|c|c|}
\hline $\mathcal{E}_{c} / T_{25}$ & $x$ & $u$ & $v$ & $w$ \\
\hline 1303.36 & 10.5984 & -1.2603 & -2.8576 & -28.0423 \\
\hline-64.2501 & -4.36718 & 19.7876 & 10.2445 & 10.4985 \\
\hline-103.454 & 3.28698 & -7.07313 & -2.08675 & -0.910597 \\
\hline-0.88015 & -0.410143 & 0.107382 & 0.0844958 & 0.361351 \\
\hline 0.212599 & -0.317509 & 1.4655 & 0.880091 & 1.08451 \\
\hline-1.0778 & -0.222653 & -0.567355 & -0.382346 & -0.536653 \\
\hline
\end{tabular}

\section{Discussion}

We solved the equation of motion of Witten's string field theory up to $O\left((1-t)^{3 / 2}\right)$ based on our ansatz using the regulated butterfly state. The leading-order solution $\left|\Psi^{(0)}\right\rangle$ gives approximately $68 \%$ of the D25-brane tension, and the two numerical solutions at the next-to-leading order give approximately $88 \%$ and $109 \%$ of the D25-brane tension, respectively. These values for the energy density are close to those obtained by level truncation up to level 2 without gauge fixing [39, 40]. Since we have studied only the first two orders of our approximation scheme, it is premature to speculate whether or not our solution will converge to the exact solution as we increase the order of the approximation, but we regard the results we have obtained so far as encouraging.

We found two solutions at the next-to-leading order, and we expect that the number of solutions will increase as we improve our ansatz by taking into account higher-order terms. Since we are solving an equation of motion, gauge fixing is not necessary. But in level truncation, the convergence of the energy density is generally better when we impose a gauge-fixing condition [39, 40]. In particular, it is known from experience that the Siegel gauge condition works well. It would be therefore interesting to incorporate gauge fixing into our approach. We should note, however, that the leading-order solution $\left|\Psi^{(0)}\right\rangle$ does not satisfy the Siegel gauge condition. Furthermore, all of the four 
terms of $\left|\Psi^{(2)}\right\rangle$ in (1.10) are necessary to have a nontrivial solution to the set of the four equations (4.33), (4.34), (4.35), and (4.36) so that we do not have any obvious ways to incorporate gauge fixing into our approach.

The idea to solve the equation of motion of Witten's string field theory using a staralgebra projector is not new, and in fact it was the original motivation to construct a star-algebra projector in [53]. It is also natural in the half-string picture $[54,55,56,57$, 58] or in the Moyal star formulation of string field theory [33, 34, 59, 35, 36, 37, 32, 38]. In a sense, our ansatz can be regarded as a well-defined way to regularize the subtleties at the open-string midpoint in the half-string picture. The wave functional of the class of string fields $\left|B_{t}(\mathcal{O})\right\rangle$ approximately factorizes into the left and right halves as $t$ approaches 1 , and the degree of freedom at the open-string midpoint is taken into account by the operator insertion $\mathcal{O}$. We can also consider more general ways to insert operators into the regulated butterfly state. For example, we can consider multiple operator insertions. As was shown in [31], the twisted regulated butterfly state can be represented in this way. We can further smear out the operator insertions along the boundary. This kind of generalization would be one possible direction to be explored in the future.

As we have seen in Section 3, our approach is applicable not only to Witten's string field theory but also to vacuum string field theory. It may also be useful in constructing a solution which has a well-defined expression for the energy density in string field theory with a different class of kinetic operators constructed in [16] and studied in $[18,60,61,22,24]$.

\section{Acknowledgments}

I would like to thank Wati Taylor and Barton Zwiebach for useful discussions. This work was supported in part by the DOE grants DF-FC02-94ER40818 (MIT) and DEFG03-92ER40701 (Caltech), and by a McCone Fellowship in Theoretical Physics from California Institute of Technology. 


\section{Appendix A. Conformal field theory formulation of string field theory}

In the CFT formulation of string field theory [45, 46], an open string field is represented as a wave functional obtained by a path integral over a certain region in a Riemann surface. For example, a state $|\phi\rangle$ in the Fock space can be represented as a wave functional on the arc $|\xi|=1$ in an upper-half complex plane of $\xi$ by path-integrating over the interior of the upper half of the unit disk $|\xi|<1$ with the corresponding operator $\phi(0)$ inserted at the origin and with the boundary condition of the open string imposed on the part of the real axis $-1 \leq \xi \leq 1$. A more general class of states such as the regulated butterfly state can be defined by a path integral over a different region of a Riemann surface with a boundary and with possible operator insertions. When we parametrize the open string on the arc as $\xi=e^{i \theta}$ with $0 \leq \theta \leq \pi$, we refer to the region $\pi / 2 \leq \theta \leq \pi$ as the left half of the open string, and to the region $0 \leq \theta \leq \pi / 2$ as the right half of the open string. We also refer to the point $\theta=\pi / 2$ as the open-string midpoint.

We use the standard definitions [2] of the inner product $\left\langle\phi_{1} \mid \phi_{2}\right\rangle$ and the star product $\left|\phi_{1} * \phi_{2}\right\rangle$. The state $\left|\phi_{1} * \phi_{2}\right\rangle$ is defined by gluing together the right half of the open string of $\left|\phi_{1}\right\rangle$ and the left half of the open string of $\left|\phi_{2}\right\rangle$. Gluing can be made by conformal transformations which map the two regions to be glued together into the same region. The inner product $\left\langle\phi_{1} \mid \phi_{2}\right\rangle$ is defined by gluing the left and right halves of the open string of $\left|\phi_{1} * \phi_{2}\right\rangle$.

We use the doubling trick throughout the paper. For example, $b c$ ghosts on an upper-half plane are extended to the lower-half plane by $c(\bar{z})=\tilde{c}(z)$ and $b(\bar{z})=\tilde{b}(z)$. The normalization of correlation functions is given by

$$
\left\langle c\left(z_{1}\right) c\left(z_{2}\right) c\left(z_{3}\right)\right\rangle=\left(z_{1}-z_{2}\right)\left(z_{1}-z_{3}\right)\left(z_{2}-z_{3}\right) \int d^{26} x
$$

In this paper, we only consider correlation functions which are independent of spacetime coordinates so that the space-time volume always factors out. We use the subscript density to denote a quantity divided by the volume factor of space-time. We use this notation for both inner products of string fields and CFT correlation functions:

$$
\begin{aligned}
\left\langle\Psi_{1} \mid \Psi_{2}\right\rangle & =\int d^{26} x\left\langle\Psi_{1} \mid \Psi_{2}\right\rangle_{\text {density }} \\
\left\langle\mathcal{O}_{1}\left(z_{1}\right) \mathcal{O}_{2}\left(z_{2}\right) \cdots \mathcal{O}_{n}\left(z_{n}\right)\right\rangle & =\int d^{26} x\left\langle\mathcal{O}_{1}\left(z_{1}\right) \mathcal{O}_{2}\left(z_{2}\right) \cdots \mathcal{O}_{n}\left(z_{n}\right)\right\rangle_{\text {density }}
\end{aligned}
$$

The normalization of a state $|\phi\rangle$ in the Fock space is fixed by the condition that the $S L(2, R)$-invariant vacuum $|0\rangle$ corresponds to the identity operator. From the normalization of correlation functions (A.1) and the standard mode expansion on a 
unit circle

$$
c_{n}=\oint \frac{d z}{2 \pi i} z^{n-2} c(z), \quad b_{n}=\oint \frac{d z}{2 \pi i} z^{n+1} b(z),
$$

the normalization of the inner product is then fixed as follows:

$$
\left\langle 0\left|c_{-1} c_{0} c_{1}\right| 0\right\rangle_{\text {density }}=1
$$

\section{Appendix B. $\left\langle\Psi^{(2)} \mid \Psi^{(2)} * \Psi^{(2)}\right\rangle$ for an arbitrary $t$}

The explicit expression of $\left\langle\Psi^{(2)} \mid \Psi^{(2)} * \Psi^{(2)}\right\rangle$ for an arbitrary $t$ is given by

$$
\begin{aligned}
& \left\langle\Psi^{(2)} \mid \Psi^{(2)} * \Psi^{(2)}\right\rangle_{\text {density }}=-\frac{81 \sqrt{3} \tilde{x}^{3}}{64}-\frac{9 \sqrt{3}\left(22+81 t^{2}\right) \tilde{x}^{2} \tilde{u}}{64} \\
& -\frac{\sqrt{3}\left(2+27 t^{2}\right)\left(38+81 t^{2}\right) \tilde{x} \tilde{u}^{2}}{64}-\frac{\sqrt{3}\left(2+3 t^{2}\right)\left(2+27 t^{2}\right)^{2} \tilde{u}^{3}}{64} \\
& +\frac{117 \sqrt{3}\left(10+27 t^{2}\right) \tilde{x}^{2} \tilde{v}}{128}+\frac{13\left(10+27 t^{2}\right)\left(22+81 t^{2}\right) \tilde{x} \tilde{u} \tilde{v}}{64 \sqrt{3}} \\
& +\frac{13\left(2+27 t^{2}\right)\left(10+27 t^{2}\right)\left(38+81 t^{2}\right) \tilde{u}^{2} \tilde{v}}{1152 \sqrt{3}} \\
& -\frac{13\left(2324+7020 t^{2}+9477 t^{4}\right) \tilde{x} \tilde{v}^{2}}{256 \sqrt{3}} \\
& -\frac{13\left(22+81 t^{2}\right)\left(2324+7020 t^{2}+9477 t^{4}\right) \tilde{u} \tilde{v}^{2}}{6912 \sqrt{3}} \\
& +\frac{13\left(167608+815724 t^{2}+1232010 t^{4}+1108809 t^{6}\right) \tilde{v}^{3}}{13824 \sqrt{3}} \\
& -\frac{9 \sqrt{3}\left(8+27 t^{2}\right) \tilde{x}^{2} \tilde{w}}{32}+\frac{3 \sqrt{3}\left(8-81 t^{2}\right)\left(2+3 t^{2}\right) \tilde{x} \tilde{u} \tilde{w}}{16} \\
& +\frac{\left(38+81 t^{2}\right)\left(592-810 t^{2}-2187 t^{4}\right) \tilde{u}^{2} \tilde{w}}{864 \sqrt{3}}+\frac{13\left(8+27 t^{2}\right)\left(10+27 t^{2}\right) \tilde{x} \tilde{v} \tilde{w}}{32 \sqrt{3}} \\
& -\frac{13\left(2+3 t^{2}\right)\left(10+27 t^{2}\right)\left(8-81 t^{2}\right) \tilde{u} \tilde{v} \tilde{w}}{96 \sqrt{3}} \\
& -\frac{13\left(8+27 t^{2}\right)\left(2324+7020 t^{2}+9477 t^{4}\right) \tilde{v}^{2} \tilde{w}}{3456 \sqrt{3}} \\
& -\frac{\sqrt{3}\left(4+9 t^{2}\right)\left(4+27 t^{2}\right) \tilde{x} \tilde{w}^{2}}{16}+\frac{\left(4+27 t^{2}\right)\left(376-1566 t^{2}-2187 t^{4}\right) \tilde{u} \tilde{w}^{2}}{432 \sqrt{3}} \\
& +\frac{13\left(4+9 t^{2}\right)\left(4+27 t^{2}\right)\left(10+27 t^{2}\right) \tilde{v} \tilde{w}^{2}}{288 \sqrt{3}}-\frac{\sqrt{3} t^{2}\left(4+9 t^{2}\right)^{2} \tilde{w}^{3}}{8} \text {. }
\end{aligned}
$$




\section{References}

[1] A. Sen and B. Zwiebach, "Tachyon condensation in string field theory," JHEP 0003, 002 (2000) [arXiv:hep-th/9912249].

[2] E. Witten, "Noncommutative Geometry And String Field Theory," Nucl. Phys. B 268, 253 (1986).

[3] N. Moeller and W. Taylor, "Level truncation and the tachyon in open bosonic string field theory," Nucl. Phys. B 583, 105 (2000) [arXiv:hep-th/0002237].

[4] R. de Mello Koch, A. Jevicki, M. Mihailescu and R. Tatar, "Lumps and p-branes in open string field theory," Phys. Lett. B 482, 249 (2000) [arXiv:hep-th/0003031].

[5] N. Moeller, A. Sen and B. Zwiebach, "D-branes as tachyon lumps in string field theory," JHEP 0008, 039 (2000) [arXiv:hep-th/0005036].

[6] W. Taylor, "A perturbative analysis of tachyon condensation," JHEP 0303, 029 (2003) [arXiv:hep-th/0208149].

[7] D. Gaiotto and L. Rastelli, "Experimental string field theory," JHEP 0308, 048 (2003) [arXiv:hep-th/0211012].

[8] W. Taylor and B. Zwiebach, "D-Branes, Tachyons, and String Field Theory," arXiv:hep-th/0311017.

[9] V. A. Kostelecky and S. Samuel, "On A Nonperturbative Vacuum For The Open Bosonic String," Nucl. Phys. B 336, 263 (1990).

[10] A. Sen, "Universality of the tachyon potential," JHEP 9912, 027 (1999) [arXiv:hep-th/9911116].

[11] A. Sen, "Descent relations among bosonic D-branes," Int. J. Mod. Phys. A 14, 4061 (1999) [arXiv:hep-th/9902105].

[12] G. T. Horowitz, J. Lykken, R. Rohm and A. Strominger, "A Purely Cubic Action For String Field Theory," Phys. Rev. Lett. 57, 283 (1986).

[13] T. Takahashi and S. Tanimoto, "Wilson lines and classical solutions in cubic open string field theory," Prog. Theor. Phys. 106, 863 (2001) [arXiv:hep-th/0107046].

[14] I. Kishimoto and K. Ohmori, "CFT description of identity string field: Toward derivation of the VSFT action," JHEP 0205, 036 (2002) [arXiv:hep-th/0112169]. 
[15] J. Kluson, "Exact solutions of open bosonic string field theory," JHEP 0204, 043 (2002) [arXiv:hep-th/0202045].

[16] T. Takahashi and S. Tanimoto, "Marginal and scalar solutions in cubic open string field theory," JHEP 0203, 033 (2002) [arXiv:hep-th/0202133].

[17] J. Kluson, "Marginal deformations in the open bosonic string field theory for N D0-branes," Class. Quant. Grav. 20, 827 (2003) [arXiv:hep-th/0203089].

[18] I. Kishimoto and T. Takahashi, "Open string field theory around universal solutions," Prog. Theor. Phys. 108, 591 (2002) [arXiv:hep-th/0205275].

[19] J. Kluson, "New solution of the open bosonic string field theory," arXiv:hep-th/0205294.

[20] J. Kluson, "Time dependent solution in open bosonic string field theory," arXiv:hep-th/0208028.

[21] J. Kluson, "Exact solutions in open bosonic string field theory and marginal deformation in CFT," arXiv:hep-th/0209255.

[22] T. Takahashi, "Tachyon condensation and universal solutions in string field theory," Nucl. Phys. B 670, 161 (2003) [arXiv:hep-th/0302182].

[23] J. Kluson, "Exact solutions in SFT and marginal deformation in BCFT," JHEP 0312, 050 (2003) [arXiv:hep-th/0303199].

[24] T. Takahashi and S. Zeze, "Gauge fixing and scattering amplitudes in string field theory around universal solutions," Prog. Theor. Phys. 110, 159 (2003) [arXiv:hep-th/0304261].

[25] D. Ghoshal and A. Sen, "Normalisation of the background independent open string field theory action," JHEP 0011, 021 (2000) [arXiv:hep-th/0009191].

[26] Y. Okawa, "Open string states and D-brane tension from vacuum string field theory," JHEP 0207, 003 (2002) [arXiv:hep-th/0204012].

[27] J. Polchinski, String Theory, Vol. I: An introduction to the bosonic string, Cambridge University Press, UK (1998).

[28] D. Gaiotto, L. Rastelli, A. Sen and B. Zwiebach, "Ghost structure and closed strings in vacuum string field theory," Adv. Theor. Math. Phys. 6, 403 (2003) [arXiv:hep-th/0111129]. 
[29] M. Schnabl, "Anomalous reparametrizations and butterfly states in string field theory," Nucl. Phys. B 649, 101 (2003) [arXiv:hep-th/0202139].

[30] D. Gaiotto, L. Rastelli, A. Sen and B. Zwiebach, "Star algebra projectors," JHEP 0204, 060 (2002) [arXiv:hep-th/0202151].

[31] Y. Okawa, "Some exact computations on the twisted butterfly state in string field theory," JHEP 0401, 066 (2004) [arXiv:hep-th/0310264].

[32] I. Bars, I. Kishimoto and Y. Matsuo, "Analytic study of nonperturbative solutions in open string field theory," Phys. Rev. D 67, 126007 (2003) [arXiv:hep-th/0302151].

[33] I. Bars, "Map of Witten's * to Moyal's *," Phys. Lett. B 517, 436 (2001) [arXiv:hep-th/0106157].

[34] I. Bars and Y. Matsuo, "Associativity anomaly in string field theory," Phys. Rev. D 65, 126006 (2002) [arXiv:hep-th/0202030].

[35] I. Bars and Y. Matsuo, "Computing in string field theory using the Moyal star product," Phys. Rev. D 66, 066003 (2002) [arXiv:hep-th/0204260].

[36] I. Bars, I. Kishimoto and Y. Matsuo, "String amplitudes from Moyal string field theory," Phys. Rev. D 67, 066002 (2003) [arXiv:hep-th/0211131].

[37] I. Bars, "MSFT: Moyal star formulation of string field theory," arXiv:hep-th/0211238.

[38] I. Bars, I. Kishimoto and Y. Matsuo, "Fermionic ghosts in Moyal string field theory," JHEP 0307, 027 (2003) [arXiv:hep-th/0304005].

[39] L. Rastelli and B. Zwiebach, "Tachyon potentials, star products and universality," JHEP 0109, 038 (2001) [arXiv:hep-th/0006240].

[40] I. Ellwood and W. Taylor, "Gauge invariance and tachyon condensation in open string field theory," arXiv:hep-th/0105156.

[41] L. Rastelli, A. Sen and B. Zwiebach, "String field theory around the tachyon vacuum," Adv. Theor. Math. Phys. 5, 353 (2002) [arXiv:hep-th/0012251].

[42] L. Rastelli, A. Sen and B. Zwiebach, "Classical solutions in string field theory around the tachyon vacuum," Adv. Theor. Math. Phys. 5, 393 (2002) [arXiv:hep-th/0102112]. 
[43] L. Rastelli, A. Sen and B. Zwiebach, "Vacuum string field theory," arXiv:hep-th/0106010.

[44] L. Rastelli, A. Sen and B. Zwiebach, "Boundary CFT construction of D-branes in vacuum string field theory," JHEP 0111, 045 (2001) [arXiv:hep-th/0105168].

[45] A. LeClair, M. E. Peskin and C. R. Preitschopf, "String Field Theory On The Conformal Plane. 1. Kinematical Principles," Nucl. Phys. B 317, 411 (1989).

[46] A. LeClair, M. E. Peskin and C. R. Preitschopf, "String Field Theory On The Conformal Plane. 2. Generalized Gluing," Nucl. Phys. B 317, 464 (1989).

[47] G. Moore and W. Taylor, "The singular geometry of the sliver," JHEP 0201, 004 (2002) [arXiv:hep-th/0111069].

[48] D. J. Gross and A. Jevicki, "Operator Formulation Of Interacting String Field Theory," Nucl. Phys. B 283, 1 (1987).

[49] D. J. Gross and A. Jevicki, "Operator Formulation Of Interacting String Field Theory. 2," Nucl. Phys. B 287, 225 (1987).

[50] N. Ohta, "Covariant Interacting String Field Theory In The Fock Space Representation," Phys. Rev. D 34, 3785 (1986) [Erratum-ibid. D 35, 2627 (1987)].

[51] E. Cremmer, A. Schwimmer and C. B. Thorn, "The Vertex Function In Witten's Formulation Of String Field Theory," Phys. Lett. B 179, 57 (1986).

[52] S. Samuel, "The Ghost Vertex In E. Witten's String Field Theory," Phys. Lett. B 181, 255 (1986).

[53] V. A. Kostelecky and R. Potting, "Analytical construction of a nonperturbative vacuum for the open bosonic string," Phys. Rev. D 63, 046007 (2001) [arXiv:hep-th/0008252].

[54] L. Rastelli, A. Sen and B. Zwiebach, "Half strings, projectors, and multiple D-branes in vacuum string field theory," JHEP 0111, 035 (2001) [arXiv:hep-th/0105058].

[55] D. J. Gross and W. Taylor, "Split string field theory I," JHEP 0108, 009 (2001) [arXiv:hep-th/0105059].

[56] T. Kawano and K. Okuyama, "Open string fields as matrices," JHEP 0106, 061 (2001) [arXiv:hep-th/0105129]. 
[57] D. J. Gross and W. Taylor, "Split string field theory II," JHEP 0108, 010 (2001) [arXiv:hep-th/0106036].

[58] K. Furuuchi and K. Okuyama, "Comma vertex and string field algebra," JHEP 0109, 035 (2001) [arXiv:hep-th/0107101].

[59] M. R. Douglas, H. Liu, G. Moore and B. Zwiebach, "Open string star as a continuous Moyal product," JHEP 0204, 022 (2002) [arXiv:hep-th/0202087].

[60] N. Drukker, "Closed string amplitudes from gauge fixed string field theory," Phys. Rev. D 67, 126004 (2003) [arXiv:hep-th/0207266].

[61] N. Drukker, "On different actions for the vacuum of bosonic string field theory," JHEP 0308, 017 (2003) [arXiv:hep-th/0301079]. 